\title{
Sports experts' unique perception of time duration based on the processing principle of an integrated model of timing
}

\author{
Binbin Jia ${ }^{\text {Corresp., } 1}$, Zhongqiu Zhang ${ }^{2}$, Tian Feng ${ }^{3}$ \\ 1 Department of Psychology, Shanghai University of Sport, Shanghai, China \\ 2 Sports Psychology and Biomechanics Research Center, China Institute of Sport Science, Beijing, China \\ 3 Physical Education College of Zhengzhou University, Zhengzhou, China \\ Corresponding Author: Binbin Jia \\ Email address: jbb1126@outlook.com
}

Background. Duration perception is an essential part of our cognitive and behavioral system, helping us interact with the outside world. An integrated model of timing, which states that the perceived duration of a given stimulus is based on the efficiency of information extraction, was recently set forth to improve current understanding of the representation and judgment of time. However, the prediction from this model that more efficient information extraction results in longer perceived duration has not been tested. Thus, the aim of this study is to investigate whether sports experts, as a group of individuals with information extraction superiority in situations relevant to their sport skill, have longer duration perceptions when they view expertise-related stimuli compared with others with no expertise experience.

Methods. For this study, 81 subjects were recruited based on a prior power analysis. The sports experts group had 27 athletes with years of professional training in diving; a wrestler group and a nonathlete group, with each of these groups having 27 subjects, were used as controls. All participants completed a classic duration reproduction task for subsecond and suprasecond durations with both the diving stimuli and general stimuli involved.

Results. The divers reproduced longer durations for diving figures compared with the general figures under both the subsecond and suprasecond time ranges, while the other samples showed the opposite pattern. Furthermore, the years of training in diving were positively correlated with the magnitude of the prolonged reproduction duration when divers viewed diving stimuli. Moreover, the diver group showed a more precise duration perception in the subsecond time range for general stimuli compared with the wrestlers and nonathletes.

Conclusion. The results suggest that sports experts perceive longer duration when viewing expertiserelated stimuli compared with others with no expertise experience. 


\section{Sports experts' unique perception of time duration}

2 based on the processing principle of an integrated

3 model of timing

4

5

6 Binbin Jia ${ }^{1}$, Zhongqiu Zhang ${ }^{2}$, Tian Feng ${ }^{3}$

7

$8 \quad{ }^{1}$ Department of Psychology, Shanghai University of Sport, Shanghai, China

$9{ }^{2}$ Sports Psychology and Biomechanics Research Center, China Institute of Sport Science,

10 Beijing, China

$11{ }^{3}$ Physical Education College of Zhengzhou University, Zhengzhou, Henan, China

12

13

14

Corresponding Author:

Binbin Jia ${ }^{1}$

No.188 Changhai Road, Shanghai, 200438, China

Email address: jbb1126@outlook.com

17 


\section{Abstract}

38 Background. Duration perception is an essential part of our cognitive and behavioral system,

39

40

41

42

43

44

45

46

47

48

49

50

51

52

53

54

55

56

57

58

59

60

61

62

63

64

65

66

67

68

69

70

71

72

73

74

75

helping us interact with the outside world. An integrated model of timing, which states that the perceived duration of a given stimulus is based on the efficiency of information extraction, was recently set forth to improve current understanding of the representation and judgment of time. However, the prediction from this model that more e $\square$ cient information extraction results in longer perceived duration has not been tested. Thus, the aim of this study is to investigate whether sports experts, as a group of individuals with information extraction superiority in situations relevant to their sport skill, have longer duration perceptions when they view expertise-related stimuli compared with others with no expertise experience. Methods. For this study, 81 subjects were recruited based on a prior power analysis. The sports expert group had 27 athletes with years of professional training in diving; a wrestler group and a nonathlete group, with each of these groups having 27 subjects, were used as controls. All participants completed a classic duration reproduction task for subsecond and suprasecond durations with both the diving stimuli and general stimuli involved. Results. The divers reproduced longer durations for diving stimuli compared with the general stimuli under both the subsecond and suprasecond time ranges, while the other samples showed the opposite pattern. Furthermore, the years of training in diving were positively correlated with the magnitude of the prolonged reproduction duration when divers viewed diving stimuli. Moreover, the diver group showed a more precise duration perception in the subsecond time range for general stimuli compared with the wrestlers and nonathletes. Conclusion. The results suggest that sports experts perceive longer duration when viewing expertise-related stimuli compared with others with no expertise experience.

\section{Introduction}

The experience of time is fundamental for how we make sense of the world. Duration perception, a component of time perception, is a "basic unit of ability" on which other cognitive and behavioral functions are based (Allman \& Meck 2012; Buhusi \& Meck 2005; Mauk \& Buonomano 2004). Due to the importance of duration perception, it has been intensively studied for many centuries (Hancock \& Block 2012), and various models have emerged to explore the underlying mechanism (Buonomano et al. 2009; Buonomano \& Maass 2009; Eagleman \& Pariyadath 2009; Matell \& Meck 2004; Noguchi \& Kakigi 2006; Treisman 1963; Walsh 2003). However, no single model is sufficient to explain all facets of duration perception (Ivry \& Schlerf 2008; Matthews \& Meck 2016). Therefore, an integrated framework combining the strength of different models will undoubtedly be welcome.

Matthews \& Meck (2016) integrated the current findings and models in the duration perception domain to provide an integrated model called the processing principle. The authors suggested that perceived duration is positively related to perceptual vividity and the ease of extracting information from stimuli. In other words, the greater the amount of information that is extracted 
76 from a given stimulus, the longer the perceived duration. Based on this proposal, sports experts, 77 as a group with especially efficient information extraction in expertise-related tasks owing to 78 their well-researched cognitive advantage (Ericsson \& Kintsch 1995; Ericsson \& Lehmann 1996; 79 Feng et al. 2017; He et al. 2018; Wei \& Luo 2010; Yarrow et al. 2009), should perceive a longer 80 duration than people who lack such expertise when they view an expertise-related stimulus.

81 However, this prediction has not yet been tested. Moreover, although some studies have 82 suggested that sports experts have more precise and stable duration perception than nonathletes 83 (Chen \& Cesari 2015; Chen et al. 2013; Chen et al. 2014), whether sports experts perceive a 84 longer duration than others for expertise-related stimuli remains unclear.

85

86

87

88

89

90

91

92

93

94

95

96

97

98

99

100

101

102

103

104

105

106

107

108

109

110

111

112

113

114

115

The present study aims to investigate sports experts' duration perception for both expertiserelated stimuli and general stimuli. Thus, we recruited divers, wrestlers and nonathletes to complete a classic duration reproduction task to evaluate the participants' duration perception of expertise-related stimuli (diving movements). General stimuli (geometric figures) were presented during the task to obtain a baseline measure. We chose divers as participants for two reasons. First, divers in China compete at a high level (Wei \& Luo 2010), which makes them suitable to serve as representative sports experts. Second, duration perception is very important for divers, since they need to perform complex movements in situations with high time pressure. Wrestlers with no diving experience but long-term physical training were recruited as a control group, and nonathletes with no diving experience or long-term physical training experience were also recruited as a control group. Moreover, duration perception in both the subsecond and suprasecond time ranges was examined during this study. Previous studies have suggested that the suprasecond time range is affected more than the subsecond range by cognition (Hayashi et al. 2014; Lewis \& Miall 2003a; Lewis \& Miall 2003b; Rammsayer \& Ulrich 2011; Rammsayer $\&$ Troche 2014). Therefore, we assume that sports experts' information extraction advantage will be expressed more in the suprasecond time range than in the subsecond time range, which is mainly ruled by automatic processes. Our specific hypothesis is that divers, compared with wrestlers and nonathletes, will show lengthened duration perception for diving-related stimuli in the suprasecond time range.

\section{Materials and Methods}

This study received approval from the regional ethics board of the China Institute of Sport Science in China (approval number: 18-04). Written informed consent was obtained from all participants before the experiment. Importantly, all the materials involved in this research can be found at osf.io/83smd/ (i.e., the task procedure, stimuli, participants' demographic information, raw data, and statistical results).

\section{Participants}

The sample size of this study was set at 81 according to an a priori power analysis using G*Power software (Franz et al. 2007). Specifically, four effects (2 main effects and 2 interaction 
116 effects) based on our hypotheses and previous studies were examined in this study. The power 117 calculations for these effects were based on the F-test (repeated-measures ANOVA); the $\alpha$ value 118 was set at 0.05 , the statistical power $(1-\beta)$ was set at 0.8 , the correlation among repeated 119 measures was set at 0.5 , and the nonsphericity correction was set at 1 . The effect size $f$ for the

120

121

122

123

124

125

126

127

128

129

130

131

132

133

134

135

136

137

138

139

140

141

142

143

144

145

146

147

148

149

150

151

152

153

154

155 effects that have previously been explored was determined according to previous studies (Chen \& Cesari 2015; Chen et al. 2013), and a small effect size was assumed based on Cohen's approaches for unexplored effects (Cohen 1988). Moreover, due to considerations of publication bias and a tendency to overestimate effect size in underpowered studies (Schafer \& Schwarz 2019; Schweizer \& Furley 2016), the effect size in our power analysis was half the magnitude of the original effects based on the findings from Open Science Collaboration (Open Science 2015). Ultimately, the largest sample size that came from these calculations was chosen to conduct the experiment. The sample size determination process is shown in Fig. 1.

The sports expert group had 27 divers (15 female, years of training 8.06 \pm 2.84 , age $14 \pm 3.09$ ) based on the criteria of being at least National Level 2 athletes (ranking among the top 3 in provincial competitions) and actively participating in national-level diving competitions. The amateur athletes group contained 27 wrestlers ( 3 female, years of training $2.94 \pm 1.63$, age $16.52 \pm 1.22)$ from a sports academy, all of them were active in provincial-level competitions. The nonathlete group consisted of 27 junior school students (13 female, age 12.56 \pm 0.51 ), none of them had any experience in sports at a competitive level. All participants had normal or corrected-to-normal vision and had no knowledge of the purpose of the experiment.

\section{Task}

In the current study, a classic duration reproduction task was utilized (Chen \& Cesari 2015). During the task, the participants were first presented with a fixation target; then, a visual stimulus was shown for a specific duration. The participants were told to remember the duration of the stimulus, and after a brief delay, they were to press and hold the space bar of a computer keyboard for the same duration using their index finger (Fig. 2).

\section{Materials and Procedures}

Two types of stimuli were employed in this task: general stimuli and expertise-related stimuli. The general stimuli, including a square, a circle, a star, and a triangle, were created in Adobe Illustrator CS6 (Adobe Systems Incorporated, San Jose, CA, USA); each one was $27 \mathrm{~mm}$ tall, and all of them were black in color (RGB: 0, 0, 0). The expertise-related stimuli (four diving movements), created with the help of four Chinese national divers, were selected from videos recorded with a Sony camera (HXR-NX3) during the divers' regular practice at the national diving pool facility. The initial images were then re-edited using Adobe Illustrator CS6 to remove the background. Different stimuli were used to quantify the anticipation and adaptation effects for duration perception (Matthews \& Meck 2016). In addition, six different durations were used for the stimulus presentations: $300 \mathrm{~ms}, 500 \mathrm{~ms}$, and $700 \mathrm{~ms}$ for the subsecond time 
156

157

158

159

160

161

162

163

164

165

166

167

168

169

170

171

172

173

174

175

176

177

178

179

180

181

182

183

184

185

186

187

188

189

190

191

192

193

194

195

196

range and $1300 \mathrm{~ms}, 1500 \mathrm{~ms}$ and $1700 \mathrm{~ms}$ for the suprasecond time range. All stimuli used in the task are shown in Fig. 3.

The task was designed and run using E-Prime 1.1 (Psychology Software Tools, Pittsburgh, PA, USA). A laptop with a 15.6-inch screen $(1024 \times 768,59 \mathrm{~Hz})$ was used to run the experimental procedure. The experiment was conducted in a room isolated from external lights and noises. The participants were seated in front of the laptop, approximately $70 \mathrm{~cm}$ away from the screen, and were given instructions for the task. The subjects were also told that counting strategies were not allowed during the task (Rattat \& Droit-Volet 2012). Then, the subjects performed a short practice session with feedback (12 trials) to ensure that they fully understood the task. After practice, the subjects were prompted to initialize the formal experiment, in which no feedback was given. The formal task was separated into 2 sessions, one for the general stimuli and another for the expertise-related stimuli. Each session had 3 blocks interspersed with 2 short breaks. Each block had 24 trials ( 4 stimuli $\times 6$ durations). The order of the combinations of stimuli and durations was pseudorandomized during each block. The participants were given a 5-minute break when the first session was completed. The order of the sessions was counterbalanced within the subjects. All participants were rewarded with gifts when the experiment was completed. The entire experimental process is presented in Fig. 4.

\section{Data Analysis and Statistics}

The raw data were fed into R (www.R-project.org) for preprocessing and plotting (RCoreTeam 2018). Six participants ( 3 from the wrestler group, 3 from the nonathlete group) were removed from further analysis because they misunderstood the task or were unwilling to cooperate (more than $50 \%$ of reproduction durations $\leq 300 \mathrm{~ms}, 73.6 \%$ overall), and six participants were added as replacements to achieve the predetermined sample size. Individuals with reaction times greater than 2000 ms during the reproduction phase were excluded (3.4\% of the data were rejected). Then, the medians for every participant under different conditions were calculated for subsequent statistical analysis. The raw data for the different groups were converted to raincloud plots to obtain robust data visualization (Allen et al. 2018).

The dependent variables were as follows: (1) The reproduction duration (RD) was used to investigate the participants' duration perception for different conditions during the task.

(2) The reproduction duration difference (RDD), defined as the RD for expertise-related stimuli minus the RD for general stimuli (baseline measure), was used to remove interindividual differences during the task. (3) Estimation bias (hereafter referred to as bias) was calculated based on the underestimation percentage (the percentage of trials for which stimulus duration $\mathrm{RD}<0)$ in the task. (4) The coefficient of variation $(\mathrm{CV})$ of the RD, based on the ratio of the standard deviation to the mean, was used to investigate the participants' duration perception stability. (5) The absolute error in the ratio (AE ratio) to the corresponding stimulus duration was used to evaluate the precision of the participants' duration perception. The RD, RDD and bias help us answer the question of whether divers perceive longer durations than wrestlers or 
197

198

199

200

201

202

203

204

205

206

207

208

209

210

211

212

213

214

215

216

217

218

219

220

221

222

223

224

225

226

227

228

229

230

231

232

233

234

235

236

nonathletes for stimuli depicting diving movements. If so, the RDD of the divers will be larger than that of the wrestlers and nonathletes in the suprasecond time range, and the bias of the divers will be smaller (less underestimated) when they view diving movement stimuli than general stimuli, while no such difference will appear in wrestlers or nonathletes. The CV and AE ratio are targeted here to replicate the findings from previous studies, which suggest that sports experts have more accurate duration perception (a smaller $\mathrm{CV}$ and $\mathrm{AE}$ ratio) than nonathletes.

The statistical analyses were conducted using JASP 0.10.0.0 (www.jasp-stats.org). A two-way repeated-measures ANOVA (with group as the between-subjects factor, time range as the withinsubjects factor, and age as the covariate) was used for the RDD. In addition, a three-way repeated-measures ANOVA (with group as the between-subjects factor, time range and image type as within-subjects factors, and age as the covariate) was employed for the $\mathrm{RD}$, bias, $\mathrm{CV}$, and AE ratio. For each of the statistical tests involved, the $95 \%$ confidence interval $(\mathrm{CI})$ for the effect size (partial $\eta^{2}$ in the F-test and Cohen's $d$ in the t-test), the Bayesian factor $\left(\mathrm{BF}_{10}\right)$ and the $\mathrm{p}$ value are provided. The Bayesian factors were calculated via the Bayesian statistics functions in JASP to quantify the strength of evidence for both the null hypothesis and the alternative hypothesis (Wagenmakers et al. 2018). The prior of the Bayes factor ANOVA is a default distribution in JASP and combines the multivariate and independent Cauchy distributions (Rouder et al. 2012), and the model parameters for the Bayes factor ANOVA are $r$ scale for fixed effects $=0.5, \mathrm{r}$ scale for random effects $=1, \mathrm{r}$ scale for covariates $=0.354$. Additionally, a uniform distribution prior for Bayesian Correlation Matrix with stretched beta prior width=1. Finally, to enable other researchers to replicate the statistical results, we provide the full statistical results of the Bayes factor ANOVA at osf.io/83smd.

More importantly, we also ran statistical analyses considering the difference in years of training between the divers and wrestlers $(8.06 \pm 2.84$ versus $2.94 \pm 1.63)$ and the gender difference between the wrestlers (only 3 females) and the other groups. The main aim was to evaluate whether our results were influenced by those factors. Therefore, we analyzed the relationship of years of training with RD and RDD in both divers and wrestlers. Moreover, we selected subsets of divers $(\mathrm{n}=10)$ and wrestlers $(\mathrm{n}=10)$ with comparable durations of training $(5.3 \pm 1.57$ versus $4.55 \pm 1.52, t(18)=1.09, p=0.291$, Cohen's $d=0.486$ ) to examine the group difference with regard to the RDD results. Additionally, to evaluate the effect of gender difference on our results, we ran repeated-measures ANOVAs of $\mathrm{RD}, \mathrm{RDD}$, bias, $\mathrm{CV}$, and $\mathrm{AE}$ ratio results for divers (15 female) and non-athletes ( 13 female) with both group and gender as the between-subjects factors, time range as the within-subjects factor, and age as the covariate.

\section{Results}

\section{RD}

The three-way repeated-measures ANOVA detected an interaction between image type and group, $F(2,77)=4.066, \mathrm{p}=0.021$, partial $\eta^{2}=0.096(90 \% \mathrm{CI}=0.008 \sim 0.192)$, and $\mathrm{BF}_{10}=2.63$. The

Peer) reviewing PDF | (2019:10:41825:1:1:NEW 12 Jan 2020) 
237

238

239

240

241

242

243

244

245

246

247

248

249

250

251

252

253

254

255

256

257

258

259

260

261

262

263

264

265

266

267

268

269

270

271

272

273

274

275 CV

divers reproduced longer RDD for expertise-related stimuli compared with general stimuli in both the subsecond and suprasecond time ranges, while the wrestlers and the controls showed the opposite result. (Fig. 5D-F). The group-time range-image type interaction did not reach the significance level $(\alpha=0.05), F(2,77)=0.556, \mathrm{p}=0.576$, partial $\eta^{2}=0.014(90 \% \mathrm{CI}=0.000 \sim 0.066)$, and $\mathrm{BF}_{10}=0.132\left(\mathrm{BF}_{01}=7.576\right)$.

\section{RDD}

The two-way repeated-measures ANOVA demonstrated a main effect of group in terms of the RDD, $F(2,77)=4.066, \mathrm{p}=0.021$, partial $\eta^{2}=0.096(90 \% \mathrm{CI}=0.008 \sim 0.192)$, and $\mathrm{BF}_{10}=1.428$. The results showed that compared with the other two groups, the divers had increased RDD values in both the subsecond and suprasecond time ranges (Fig. 6E). A comparison of the three groups revealed the following results with regard to the divers and wrestlers: $t=2.830$, Cohen's $d=0.314$ $(95 \% \mathrm{CI}=0.213 \sim 1.320), \mathrm{p}_{\mathrm{holm}}=0.018$, and $\mathrm{BF}_{10}=11.668$. The results of the comparison between the divers and nonathlete controls were as follows: $\mathrm{t}=1.132$, Cohen's $\mathrm{d}=0.126(95 \% \mathrm{CI}=-$ $0.230 \sim 0.843)$, $\mathrm{p}_{\text {holm }}=0.261$, and $\mathrm{BF}_{10}=1.646$.

\section{RDD and Years of Diving Training}

To explore the relationship between the RDD and years of diving training among the divers, we used the Pearson correlation coefficient. The results showed that in the subsecond time range, $\mathrm{r}=0.315(95 \% \mathrm{CI}=-0.083 \sim 0.626), \mathrm{p}=0.117$, and $\mathrm{BF}_{10}=0.780\left(\mathrm{BF}_{01}=1.282\right)$; additionally, in the suprasecond time range, $\mathrm{r}=0.589(95 \% \mathrm{CI}=0.262 \sim 0.795), \mathrm{p}=0.002$, and $\mathrm{BF}_{10}=28.127$. The longer the divers' training experience, the larger their RDD, especially in the suprasecond trials. The raw data are shown in Fig. 7. One diver's data were excluded from statistical analysis because of their extreme value ( $<$ mean $-3.5 * \mathrm{SD}$ in the suprasecond condition); the results including this extreme value are $\mathrm{r}=0.198(95 \% \mathrm{CI}=-0.197 \sim 0.538), \mathrm{p}=0.322$, and $\mathrm{BF}_{10}=0.381\left(\mathrm{BF}_{01}=2.625\right)$ in the subsecond time range and $\mathrm{r}=0.283(95 \% \mathrm{CI}=-0.108 \sim 0.599), \mathrm{p}=0.152$, and $\mathrm{BF}_{10}=0.633\left(\mathrm{BF}_{01}\right.$ $=1.58)$ in the suprasecond time range.

\section{Bias}

As shown in Fig. 8A-C, the three-way repeated-measures ANOVA detected a main effect of time range, $F(1,77)=8.866, \mathrm{p}=0.004$, partial $\eta^{2}=0.103(90 \% \mathrm{CI}=0.020 \sim 0.218)$, and $\mathrm{BF}_{10}=$

$8.954 \mathrm{e}+59$, with most of the participants in all three groups overestimating (bias $<0.5)$ more at subsecond durations and underestimating (bias $>0.5$ ) more at suprasecond durations. Moreover, an interaction effect between imagetype and group was found, $F(2,77)=5.338, \mathrm{p}=0.007$, partial $\eta^{2}=0.122(90 \% \mathrm{CI}=0.021 \sim 0.228)$, and $\mathrm{BF}_{10}=1.963$ (Fig. 8D). This interaction effect revealed that the divers overestimated more in the diving image condition than in the general image condition, while the other participants showed the opposite result. 
276 As illustrated in Fig. 9D, the three-way repeated-measures ANOVA detected an interaction of

277 time range and age (covariate), $F(1,76)=6.975, \mathrm{p}=0.010$, partial $\eta^{2}=0.084(90 \%$

$278 \mathrm{CI}=0.012 \sim 0.195)$, and $\mathrm{BF}_{10}=3.907 \mathrm{e}+20$. The results showed that the participants in all three

279 groups had a more stable reproduction duration in the suprasecond time range than in the

280 subsecond time range, $\mathrm{t}=11.597$, Cohen's $\mathrm{d}=1.297$ (95\% $\mathrm{CI}=-0.996 \sim 1.592), \mathrm{p}<0.0001$. In

281 addition, the main effect of group(Fig. 9E-F) did not reach the significance level $(\alpha=0.05), F(2$,

$28276)=0.701, \mathrm{p}=0.499$, partial $\eta^{2}=0.018(90 \% \mathrm{CI}=0.000 \sim 0.073)$, and $\mathrm{BF}_{10}=0.117\left(\mathrm{BF}_{01}=8.547\right)$,

283 nor did the interaction effect between group and time range, $F(2,76)=2.263, \mathrm{p}=0.111$, partial

$284 \eta^{2}=0.056(90 \% \mathrm{CI}=0.000 \sim 0.079)$, and $\mathrm{BF}_{10}=0.073\left(\mathrm{BF}_{01}=13.699\right)$. One wrestler's $\mathrm{CV}$ data

285 were removed from the data plot and ANOVA due to their extreme value $(>$ mean $+8 * \mathrm{SD}$ in the

286 diving image condition); the ANOVA results including this extreme value are $F(1,77)=4.751$,

$287 \mathrm{p}=0.032$, partial $\eta^{2}=0.058(90 \% \mathrm{CI}=0.002 \sim 0.159)$, and $\mathrm{BF}_{10}=1.979 \mathrm{e}+6$ for the interaction of

288 time range-age. The results for the main effect of group were $F(2,77)=0.619, \mathrm{p}=0.541$, partial

$289 \eta^{2}=0.016(90 \% \mathrm{CI}=0.000 \sim 0.068)$, and $\mathrm{BF}_{10}=0.169\left(\mathrm{BF}_{01}=5.917\right)$, and those for the interaction

290 effect between group and time range were $F(2,77)=0.831, \mathrm{p}=0.440$, partial $\eta^{2}=0.021(90 \%$

$291 \mathrm{CI}=0.000 \sim 0.082)$, and $\mathrm{BF}_{10}=0.060\left(\mathrm{BF}_{01}=16.667\right)$.

292

293

294

295

296

297

298

299

300

301

302

303

304

305

306

307

308

309

310

311

312

313

314

315

\section{AE Ratio}

The three-way repeated-measures ANOVA detected a main effect of time range (Fig. 10D), $F$ (1, $77)=6.544, \mathrm{p}=0.012$, partial $\eta^{2}=0.078(90 \% \mathrm{CI}=0.010 \sim 0.187)$, and $\mathrm{BF}_{10}=7.477 \mathrm{e}+11$, with most of the participants having a larger $\mathrm{AE}$ ratio in the subsecond time range than in the suprasecond time range. Moreover, an interaction effect of time range, image type, and group was

revealed(Fig. 10E-F), $F(2,77)=5.254, \mathrm{p}=0.007$, partial $\eta^{2}=0.120(90 \% \mathrm{CI}=0.020 \sim 0.226)$, and $\mathrm{BF}_{10}=0.874\left(\mathrm{BF}_{01}=1.144\right)$, revealing that the diver group produced smaller $\mathrm{AE}$ ratios than the other groups in the subsecond time range under the general image condition.

\section{Effects of Years of Training and Gender}

To explore the relationship of years of training with RD and RDD in wrestlers and divers, we used the Pearson correlation coefficient. The results showed no evidence that years of training affected RD for diving images in wrestlers or divers (Fig. 11A-B). Specifically, $\mathrm{r}=-0.107$ (95\% $\mathrm{CI}=-0.468 \sim 0.285), \mathrm{p}=0.596$ in the subsecond time range and $\mathrm{r}=-0.055(95 \% \mathrm{CI}=-0.426 \sim 0.332)$, $\mathrm{p}=0.785$ in the suprasecond time range for wrestlers; $\mathrm{r}=-0.207(95 \% \mathrm{CI}=-0.55 \sim 0.196), \mathrm{p}=0.31$ in the subsecond time range and $\mathrm{r}=-0.059(95 \% \mathrm{CI}=-0.437 \sim 0.336), \mathrm{p}=0.774$ in the suprasecond time range for divers. Additionally, there was no evidence supporting a relationship between years of training and RDD in wrestlers (Fig. 11C). We found that $\mathrm{r}=-0.011, \mathrm{p}=0.957(95 \% \mathrm{CI}=-$ $0.389 \sim 0.371$ ) in the subsecond time range and $\mathrm{r}=0.153(95 \% \mathrm{CI}=-0.241 \sim 0.503), \mathrm{p}=0.447$ in the suprasecond time range for the wrestlers. More importantly, the relationship between years of training and RDD increased for divers when we considered only expertise-related training experience (Fig. 11D-E). For example, one diver had 8 years of training experience, including 3 years of training for basketball and 5 years of training for diving. When we ran the analysis 
316 between years of diving training and RDD, $\mathrm{r}$ increased from 0.133 (all training) to 0.315 (only

317 diving training) in the subsecond time range and increased from 0.433 to 0.589 in the

318 suprasecond time range. Finally, the RDD group difference remained (divers $>$ wrestlers) when

319 we compared participants in both groups with compatible lengths of training (Fig. 11F-G).

320 Specifically, the statistical results were $\mathrm{t}(18)=1.807, \mathrm{p}=0.026$, Cohen's $\mathrm{d}=1.087(95 \%$

$321 \mathrm{CI}=0.127 \sim 2.016)$ in the subsecond time range and $\mathrm{t}(18)=2.423, \mathrm{p}=0.026$, Cohen's $\mathrm{d}=0.95(95 \%$

$322 \mathrm{CI}=-0.011 \sim 1.869)$ in the suprasecond time range.

323

324

325

326

327

328

329

330

331

332

333

334

335

336

337

338

339

340

341

342

343

344

345

346

347

348

349

350

351

352

353

354

355

Finally, ANOVA with an effect of gender revealed statistically interactions of time range and gender on bias $(F(1,48)=6.381, \mathrm{p}=0.015)$ and AE ratio $(F(1,48)=4.37, \mathrm{p}=0.042)$. However, no other results (whether main effects or interactions) reached the significance level $(\alpha=0.05)$ in $\mathrm{RD}, \mathrm{RDD}$ and $\mathrm{CV}$.

\section{Discussion}

The main purpose of this study was to investigate the duration perception of sports experts. Based on the processing principle, the initial prediction was that the divers would reproduce longer durations than wrestlers or nonathletes for expertise-related stimuli (diving movements) in the suprasecond time range. The RD, RDD and bias results are consistent with this hypothesis.

According to the RD and RDD results, the divers reproduced longer durations when viewing diving movements than general stimuli in both subsecond and suprasecond ranges, while the wrestlers and nonathletes showed the opposite result. First, the divers' increased duration perception for expertise-related stimuli compared with general stimuli in the suprasecond time range is in line with our prediction. We attribute this phenomenon to sports efforts' efficiency advantage in extracting information from expertise-related stimuli. Wei \& Luo (2010) found that divers utilized kinesthetic imagery more efficiently than novices for activities in which they had expertise. Sports experts' improved information extraction efficiency might stem from their cognitive advantages, which have been well demonstrated by many researchers. For instance, a review by Yarrow et al. (2009) concluded that sports experts show superior performance in perception, anticipation and decision making and that this superior performance is task specific and dependent on extensive practice. In addition, many other studies have revealed that sports experts outperform nonathletes in attention and memory for expertise-related tasks (Ericsson \& Kintsch 1995; He et al. 2018). Consequently, the advantages that sports experts hold in perception, attention and memory facilitate the extraction of information from expertise-related stimuli, causing them to perceive longer durations than nonathletes. In line with this suggestion, some studies have already found that as a result of better attention and memory, duration perception lasts longer (Baudouin et al. 2006; Seifried \& Ulrich 2011).

Meanwhile, many findings from the domain of time perception indicate that different temporal information processes and neural systems are involved in different time ranges (Hayashi et al. 
356

357

358

359

360

361

362

363

364

365

366

367

368

369

370

371

372

373

374

375

376

377

378

379

380

381

382

383

384

385

386

387

388

389

390

391

392

393

394

395

2014; Lewis \& Miall 2003a; Lewis \& Miall 2003b; Rammsayer \& Ulrich 2011), specifically, a cognitively controlled mechanism to process durations in the suprasecond range and a sensory or automatic mechanism to process durations in the subsecond range. For example, Hayashi et al. (2014) found that subsecond durations are processed in the motor system, whereas suprasecond durations are processed in the parietal cortex using attention and working memory. Therefore, combined with athletes' cognitive advantages in expertise-related tasks and the larger role of cognition at longer durations, the increase in the perceived duration of suprasecond diving stimuli compared with general stimuli among divers is quite reasonable based on the processing principle, which suggests that more efficient information extraction for a given stimulus results in a longer perceived duration.

More interestingly, the RD and RDD results also indicated that the divers reproduced a longer duration for expertise-related stimuli than for general stimuli in the subsecond time range, which is believed to be dominated by automatic processing. This result is beyond our initial prediction, since the effect of cognition on processing is smaller at subsecond durations than at suprasecond durations. However, Rammsayer \& Troche (2014) argued that there is no clear-cut boundary between different mechanisms at different time ranges and that it is more appropriate to proceed from the notion of a continuum with regard to the involvement of cognitive processes. Thus, the result noted above can be explained by the fact that the sports experts' intense practice improved cognition far beyond what nonexperts can attain. In fact, many studies have already argued that athletes from open-skill sports display better automatic information processing in coping with high time pressure situations than do nonathletes (Guldenpenning et al. 2015; Guldenpenning et al. 2011). Similarly, You et al. (2018) found that table tennis players outperformed nonathletes in regard to response inhibition even in an unconscious condition where the participants were unaware of the stimulus in the task. Moreover, the findings of Meng et al. (2019) also suggest that motor expertise modulates unconscious executive control and is positively correlated with unconscious executive control in table tennis players. These results, combined with the fact that divers, similar to many athletes in open-skill sports, must perform complex movements under high time pressure, suggest that the efficient information processing conferred by their cognitive advantages affects subsecond-duration processing, which is considered to be dominated by automatic or unconscious mechanisms.

In addition, the analysis of the divers' training time and RDD showed that the longer their diving training, the larger the RDD they produced, especially for suprasecond durations. This result is consistent with the suggestions of some researchers that athletes' cognitive advantage in expertise-related tasks is mainly practice dependent and cannot be attributed to innate differences (Meng et al. 2019; Yarrow et al. 2009). In the case of the divers, a longer sport-specific training period resulted in more efficient information processing with regard to expertise-related stimuli, and this more efficient information extraction led these athletes to reproduce a longer duration for expertise-related stimuli than for general stimuli. Moreover, the correlation was stronger at 
396

397

398

399

400

401

402

403

404

405

406

407

408

409

410

411

412

413

414

415

416

417

418

419

420

421

422

423

424

425

426

427

428

429

430

431

432

433

434

435

suprasecond than subsecond durations, confirming that cognitive processes are more involved in longer durations (Lewis \& Miall 2003b; Rammsayer \& Troche 2014).

It is important to note that the difference in years of training between the divers and wrestlers $(8.06 \pm 2.84$ versus $2.94 \pm 1.63)$ and the gender difference between the wrestlers (only 3 females) and the other groups might affect our interpretation of the results. However, the analysis showed no evidence that the group difference in RDD can be attributed to the difference in length of training between divers and wrestlers. For example, the analysis showed consistent results for divers and wrestlers with comparable lengths of training. More importantly, the relationship between training experience and RDD was strengthened when we considered only expertiserelated training. Therefore, we supposed that expertise-related training experience played a larger role than general physical training in explaining the increased RDD of divers compared with wrestlers. Additionally, there was little evidence supporting an effect of gender difference on the RDD results. For example, no significant effect was found in ANOVA with gender as a betweensubjects factor. Although there were 2 interactions that reached the significance level in our analysis for other dependent variables, the low positive finding rate $(2 / 32)$ combined with the lack of evidence for a gender effect on duration perception in other studies led us to believe that the current results were solid despite the gender differences between groups.

Regarding the bias results, most participants in the task showed a tendency to overestimate subsecond durations and underestimate suprasecond durations, which is consistent with the results of other studies (Chen et al. 2014). This finding is a well-known phenomenon in the field of time perception, especially in the reproduction task, and scholars have already offered a reasonable explanation (Wearden \& Lejeune 2007). However, there is also a new perspective based on the Bayesian model, which suggests that the observer integrates a noisy representation of the stimulus with prior information about the stimulus distribution to produce a posterior distribution for the duration to be judged (Jazayeri \& Shadlen 2010). More importantly, the group-image type interaction revealed that when the stimuli changed from general to diving stimuli, only the divers overestimated more in both the subsecond and suprasecond time ranges. This result combined with the RD and RDD outcomes confirmed that only the divers reproduced longer durations when they viewed expertise-related stimuli compared with general stimuli, while wrestlers and nonathletes with no diving experience showed the opposite result.

The $\mathrm{CV}$ and $\mathrm{AE}$ ratio results showed that all participants performed better as the stimulus duration grew longer (smaller $\mathrm{CV}$ and $\mathrm{AE}$ ratio in the suprasecond time range than in the subsecond time range), which is in line with the results of other studies (Chen \& Cesari 2015; Hayashi et al. 2014; Lewis \& Miall 2009). However, previous research found that sports experts have a more precise and stable timing ability than nonathletes, and the results of a reduced $\mathrm{AE}$ ratio and $\mathrm{CV}$ for sports experts were not repeated in this research. For example, Chen et al. (2014) found that compared with nonathletes, athletes had reduced AE ratios in subsecond time 
436 ranges and reduced CVs in both subsecond and suprasecond time ranges for both expertise437 related and non-expertise-related stimuli. Our results suggest that there were no differences 438 among the different groups with regard to the $\mathrm{CV}$. Regarding the AE ratio, the present study 439 revealed that the divers outperformed the wrestlers and nonathletes in the subsecond time range 440 for general stimuli. Some have argued that the superior precision of duration perception in sports 441 experts can be viewed as a result of their sharpened perceptual-motor system induced by long-

442

443

444

445

446

447

448

449

450

451

452

453

454

455

456

457

458

459

460

461

462

463

464

465

466

467

468

469

470

471

472

473

474 term training (Chen \& Cesari 2015). Indeed, long-term sports training or motor skill learning experience has been suggested as a factor that induces functional and structural changes in the human brain (Dayan \& Cohen 2011; Huang et al. 2018). Furthermore, many findings have shown that the cerebellum is affected by training experience (Cannonieri et al. 2007; Han et al. 2009; Kim et al. 2014; Park et al. 2009). Meanwhile, the cerebellum has been strongly linked to subsecond time perception (Hayashi et al. 2013; Hayashi et al. 2014; Lewis \& Miall 2003b; Wiener et al. 2010).

Therefore, one possible explanation for the reduced $\mathrm{AE}$ ratio found in the diver group is that the divers' cerebellar areas underwent changes induced by intense general physical training, which would improve timing ability in the subsecond time range. Additionally, the wrestlers showed no difference from non-athletes in terms of AE ratios, indicating that their general physical training experience might not be sufficient to upgrade their motor system for improved timing ability in the subsecond time range. Indeed, results from Sysoeva et al. (2013) revealed that elite athletes (swimmers and skiers) showed higher accuracy in a tapping task than amateur athletes (wrestlers) or nonathletes. Moreover, Kim et al. (2014) found that only elite archers showed increased activity in the cerebellar area in a simulated archery aiming task. However, the divers' advantage in timing precision disappeared in the expertise-related stimulus condition, which is also consistent with our prediction. Since the divers reproduced longer durations for diving stimuli than for general stimuli, the longer durations resulted in larger $\mathrm{AE}$ ratios.

There are two possible reasons for the inconsistency between the present results and those of previous studies. First, the task instructions for the participants in previous studies emphasized precision, which might have led different groups (athletes, nonathletes) to take advantage of different strategies (Chen \& Cesari 2015). For example, sports experts might be more likely to employ a counting strategy during the task, since the instructions did not forbid counting. This difference could account for the superior performance (i.e., reduced $\mathrm{CV}$ and $\mathrm{AE}$ ratio) of sports experts (Rattat \& Droit-Volet 2012). Second, the sample sizes in previous studies were quite small. For instance, the elite athlete group in Chen et al. (2014) had only 12 participants, and as a result, there might have been a greater proportion of false positive findings due to the problem of low statistical power (Fraley \& Vazire 2014; Schweizer \& Furley 2016). However, the lack of additional evidence from other studies examining sports experts' duration perception makes it difficult to draw solid conclusions. Therefore, although the present study showed that athletes 
475 perceive longer durations than nonexperts when viewing expertise-related stimuli, further

476 research is needed to provide more consistent evidence.

477

478

479

480

481

482

483

484

485

486

487

488

489

490

491

492

493

494

495

496

497

498

499

500

501

502

503

504

505

506

507

508

509

510

511

512

513

Finally, we would like to discuss two potential implications of the findings from this study. First, the perceived duration of expertise-related stimuli can be used to evaluate athletes' information processing efficiency. In the present study, we attributed divers' extended duration perception for expertise-related stimuli to an increased efficiency of information extraction because of their sport-specific (i.e., diving) training experience. Therefore, athletes who perceive longer durations for expertise-related stimuli are likely to have higher information processing efficiency. Information processing efficiency certainly plays a large role in athletic performance, and this inference is supported by the connection between the length of diving training and the extent to which the duration of expertise-related stimuli is overestimated in our research. Thus, the perceived duration of expertise-related stimuli might help coaches select young athletes with especially great potential. Second, general physical training might be a good supplement to other treatments to correct distortions in time perception and time performance caused by a number of different neurological and psychiatric conditions (e.g., attention-deficit/hyperactivity disorder (ADHD) and autism). Our results showed that sports experts with long-term physical training experience have superior timing ability (i.e., timing precision). This finding is consistent with the work of other researchers and indicates that long-term physical training might improve timing ability. Thus, exercise intervention for people with ADHD or autism might improve their timing ability.

\section{Limitations}

The current study has 3 limitations. First, some interpretations of our study results must be regarded with caution due to the differences in years of training and gender between the wrestlers and divers. Although our analysis confirmed that the current results and interpretations are reasonable even in light of those factors, experts with compatible lengths of training in different sports will enable researchers to precisely evaluate the effects of expertise-related training and general physical training on duration perception. Second, the duration reproduction task we employed is not a pure perception task, as the bisection and generalization tasks are. Rather, we made a simplifying assumption that the motor noise (i.e., pressing the button) was constant for all subjects. However, we obtained baseline evaluations of all participants in the general stimulus session and utilized the RDD to evaluate the difference in duration perception for different stimuli in an attempt to ensure that the main results of the present study were not excessively affected by the participants' motor noise. More importantly, the reproduction task was utilized not only because it offered a continuous measurement of duration perception but also because it enabled us to compare our results with those of previous studies. The third limitation is that the correlation analysis between the magnitude of overestimation and the length of training in the diver group was flawed because the correlation statistics were not part of our 
514 initial data-processing plan. In future studies, a more reasonable sample size based on a priori

515 power analysis is recommended.

516

\section{Conclusions}

518 The divers reproduced longer durations than wrestlers or nonathletes when they viewed

519 expertise-related stimuli in the temporal reproduction task. This outcome is consistent with our

520 prediction based on the processing principle, which hypothesized that more efficient information

521 extraction would result in longer perceived duration. In addition, the positive correlation between

522 the years of diving training and the magnitude of duration overestimation for expertise-related

523 stimuli indicated that the sports experts' advantage in information extraction is a result of

524 professional training in the relevant sport. Moreover, this finding also revealed that divers with

525 long-term athletic training experience had more precise duration perception than others in the

526 subsecond time range only for general stimuli, suggesting that intense general physical training

527 might improve timing accuracy.

528

529

530

531

532

533

534

535

536

537

538

539

540

541

542

543

544

545

546

547

548

549

550

551

552

553

554

555

556

557

558

559

\section{References}

Allen M, Poggiali D, Whitaker K, Marshall TR, and Kievit R. 2018. Raincloud plots: a multiplatform tool for robust data visualization. PeerJ Preprints 6:e27137v1 https://doi.org10.7287/peerj.preprints.27137v1

Allman MJ, and Meck WH. 2012. Pathophysiological distortions in time perception and timed performance. Brain 135:656-677. 10.1093/brain/awr210

Baudouin A, Vanneste S, Pouthas V, and Isingrini M. 2006. Age-related changes in duration reproduction: involvement of working memory processes. Brain Cogn 62:17-23. 10.1016/j.bandc.2006.03.003

Buhusi CV, and Meck WH. 2005. What makes us tick? Functional and neural mechanisms of interval timing. Nat Rev Neurosci 6:755-765. 10.1038/nrn1764

Buonomano DV, Bramen J, and Khodadadifar M. 2009. Influence of the interstimulus interval on temporal processing and learning: testing the state-dependent network model. Philos Trans R Soc Lond B Biol Sci 364:1865-1873. 10.1098/rstb.2009.0019

Buonomano DV, and Maass W. 2009. State-dependent computations: spatiotemporal processing in cortical networks. Nat Rev Neurosci 10:113-125. 10.1038/nrn2558

Cannonieri GC, Bonilha L, Fernandes PT, Cendes F, and Li LM. 2007. Practice and perfect_ length of training and structural brain changes in experienced typists. Neuroreport 18:1063-1066.

Chen YH, and Cesari P. 2015. Elite athletes refine their internal clocks. Motor Control 19:90101. 10.1123/mc.2013-0081

Chen YH, Pizzolato F, and Cesari P. 2013. Observing Expertise-Related Actions Leads to Perfect Time Flow estimations. Plos One 8:e55294. 10.1371/journal.pone.0055294.g001

Chen YH, Pizzolato F, and Cesari P. 2014. Time flies when we view a sport action. Exp Brain Res 232:629-635. 10.1007/s00221-013-3771-2

Cohen J. 1988. Statistical power analysis for the behavioral sciences, 2nd Edn. New York: NY: Academic Press.

Dayan E, and Cohen LG. 2011. Neuroplasticity subserving motor skill learning. Neuron 72:443454. 10.1016/j.neuron.2011.10.008

Eagleman DM, and Pariyadath V. 2009. Is subjective duration a signature of coding efficiency? Philos Trans R Soc Lond B Biol Sci 364:1841-1851. 10.1098/rstb.2009.0026 
560

561

562

563

564

565

566

567

568

569

570

571

572

573

574

575

576

577

578

579

580

581

582

583

584

585

586

587

588

589

590

591

592

593

594

595

596

597

598

599

600

601

602

603

604

605

606

607

608

Ericsson KA, and Kintsch W. 1995. Long-term working memory. Psychological Review 102:211245.

Ericsson KA, and Lehmann AC. 1996. Expert and exceptional performance: evidence of maximal adaptation to task constraints. Annu Rev Psychol 47:273-305. 10.1146/annurev.psych.47.1.273

Feng T, Zhang Z, Ji Z, Jia B, and Li Y. 2017. Selective Effects of Sport Expertise on the Stages of Mental Rotation Tasks With Object-Based and Egocentric Transformations. Adv Cogn Psychol 13:248-256. 10.5709/acp-0225-x

Fraley RC, and Vazire S. 2014. The N-pact factor: evaluating the quality of empirical journals with respect to sample size and statistical power. Plos One 9:e109019. 10.1371/journal.pone.0109019

Franz F, Edgar E, Albeert-Georg L, and Axel B. 2007. G*Power 3: A flexible statistical power analysis program for the social, behavioral, and biomedical sciences. Behavior Research Methods 39:175-191.

Guldenpenning I, Braun JF, Machlitt D, and Schack T. 2015. Masked priming of complex movements: perceptual and motor processes in unconscious action perception. Psychol Res 79:801-812. 10.1007/s00426-014-0607-z

Guldenpenning I, Koester D, Kunde W, Weigelt M, and Schack T. 2011. Motor expertise modulates the unconscious processing of human body postures. Exp Brain Res 213:383-391. 10.1007/s00221-011-2788-7

Han Y, Yang H, Lv Y-T, Zhu C-Z, He Y, Tang H-H, Gong Q-Y, Luo Y-J, Zang Y-F, and Dong Q. 2009. Gray matter density and white matter integrity in pianists' brain: A combined structural and diffusion tensor MRI study. Neuroscience Letters 459:3-6. 10.1016/j.neulet.2008.07.056

Hancock PA, and Block RA. 2012. The psychology of time: a view backward and forward. The American Journal of Psychology 125:267-274.

Hayashi MJ, Kanai R, Tanabe HC, Yoshida Y, Carlson S, Walsh V, and Sadato N. 2013. Interaction of numerosity and time in prefrontal and parietal cortex. J Neurosci 33:883893. 10.1523/JNEUROSCI.6257-11.2013

Hayashi MJ, Kantele M, Walsh V, Carlson S, and Kanai R. 2014. Dissociable neuroanatomical correlates of subsecond and suprasecond time perception. J Cogn Neurosci 26:16851693. 10.1162/jocn_a_00580

He M, Qi C, Lu Y, Song A, Hayat SZ, and Xu X. 2018. The Sport Expert's Attention Superiority on Skill-related Scene Dynamic by the Activation of left Medial Frontal Gyrus: An ERP and LORETA Study. Neuroscience 379:93-102. 10.1016/j.neuroscience.2018.02.043

Huang H, Wang J, Seger C, Lu M, Deng F, Wu X, He Y, Niu C, Wang J, and Huang R. 2018. Long-term intensive gymnastic training induced changes in intra- and inter-network functional connectivity: an independent component analysis. Brain Struct Funct 223:131144. 10.1007/s00429-017-1479-y

Ivry RB, and Schlerf JE. 2008. Dedicated and intrinsic models of time perception. Trends Cogn Sci 12:273-280. 10.1016/j.tics.2008.04.002

Jazayeri M, and Shadlen MN. 2010. Temporal context calibrates interval timing. Nat Neurosci 13:1020-1026. 10.1038/nn.2590

Kim W, Chang Y, Kim J, Seo J, Ryu K, Lee E, Woo M, and Janelle CM. 2014. An fMRI Study of Differences in Brain Activity Among Elite, Expert, and Novice Archers at the Moment of Optimal Aiming. Cognitive And Behavioral Neurology 27:173-182.

Lewis PA, and Miall RC. 2003a. Brain activation patterns during measurement of sub- and supra-second intervals. Neuropsychologia 41:1583-1592. 10.1016/s00283932(03)00118-0 
609

610

611

612

613

614

615

616

617

618

619

620

621

622

623

624

625

626

627

628

629

630

631

632

633

634

635

636

637

638

639

640

641

642

643

644

645

646

647

648

649

650

651

652

653

654

655

656

657

658

Lewis PA, and Miall RC. 2003b. Distinct systems for automatic and cognitively controlled time measurement: evidence from neuroimaging. Current Opinion in Neurobiology 13:250255. 10.1016/s0959-4388(03)00036-9

Lewis PA, and Miall RC. 2009. The precision of temporal judgement: milliseconds, many minutes, and beyond. Philos Trans R Soc Lond B Biol Sci 364:1897-1905. $10.1098 /$ rstb. 2009.0020

Matell MS, and Meck WH. 2004. Cortico-striatal circuits and interval timing: coincidence detection of oscillatory processes. Brain Res Cogn Brain Res 21:139-170. 10.1016/j.cogbrainres.2004.06.012

Matthews WJ, and Meck WH. 2016. Temporal cognition: Connecting subjective time to perception, attention, and memory. Psychol Bull 142:865-907. 10.1037/bul0000045

Mauk MD, and Buonomano DV. 2004. The neural basis of temporal processing. Annu Rev Neurosci 27:307-340. 10.1146/annurev.neuro.27.070203.144247

Meng F, Li A, You Y, and Xie C. 2019. Motor expertise modulates unconscious rather than conscious executive control. PeerJ 7:e6387. 10.7717/peerj.6387

Noguchi Y, and Kakigi R. 2006. Time representations can be made from nontemporal information in the brain: an MEG study. Cereb Cortex 16:1797-1808. 10.1093/cercor/bhj117

Open Science C. 2015. PSYCHOLOGY. Estimating the reproducibility of psychological science. Science 349:aac4716. 10.1126/science.aac4716

Park IS, Lee KJ, Han JW, Lee NJ, Lee WT, Park KA, and Rhyu IJ. 2009. Experience-dependent plasticity of cerebellar vermis in basketball players. Cerebellum 8:334-339. 10.1007/s12311-009-0100-1

Rammsayer T, and Ulrich R. 2011. Elaborative rehearsal of nontemporal information interferes with temporal processing of durations in the range of seconds but not milliseconds. Acta Psychol (Amst) 137:127-133. 10.1016/j.actpsy.2011.03.010

Rammsayer TH, and Troche SJ. 2014. In search of the internal structure of the processes underlying interval timing in the sub-second and the second range: a confirmatory factor analysis approach. Acta Psychol (Amst) 147:68-74. 10.1016/j.actpsy.2013.05.004

Rattat AC, and Droit-Volet S. 2012. What is the best and easiest method of preventing counting in different temporal tasks? Behav Res Methods 44:67-80. 10.3758/s13428-011-0135-3

RCoreTeam. 2018. R: A language and environment for statistical computing. R Foundation for Statistical Computing, Vienna, Austria.

Rouder JN, Morey RD, Speckman PL, and Province JM. 2012. Default Bayes factors for ANOVA designs. Journal of Mathematical Psychology 56:356-374. 10.1016/j.jmp.2012.08.001

Schafer T, and Schwarz MA. 2019. The Meaningfulness of Effect Sizes in Psychological Research: Differences Between Sub-Disciplines and the Impact of Potential Biases. Front Psychol 10:813. 10.3389/fpsyg.2019.00813

Schweizer G, and Furley P. 2016. Reproducible research in sport and exercise psychology: The role of sample sizes. Psychology of Sport and Exercise 23:114-122. 10.1016/j.psychsport.2015.11.005

Seifried T, and Ulrich R. 2011. Exogenous visual attention prolongs perceived duration. Atten Percept Psychophys 73:68-85. 10.3758/s13414-010-0005-6

Sysoeva OV, Wittmann M, Mierau A, Polikanova I, Struder HK, and Tonevitsky A. 2013. Physical exercise speeds up motor timing. Front Psychol 4:612. 10.3389/fpsyg.2013.00612

Treisman M. 1963. Temporal discrimination and the indifference interval: Implications for a model of the "internal clock". Psychological Monographs: General and Applied 77:1-31. $10.1037 / \mathrm{h} 0093864$

Peer] reviewing PDF | (2019:10:41825:1:1:NEW 12 Jan 2020) 
659

660

661

662

663

664

665

666

667

668

669

670

671

672

673

674

675

676

677

678

679

680
Wagenmakers EJ, Love J, Marsman M, Jamil T, Ly A, Verhagen J, Selker R, Gronau QF, Dropmann D, Boutin B, Meerhoff F, Knight P, Raj A, van Kesteren EJ, van Doorn J, Smira M, Epskamp S, Etz A, Matzke D, de Jong T, van den Bergh D, Sarafoglou A, Steingroever H, Derks K, Rouder JN, and Morey RD. 2018. Bayesian inference for psychology. Part II: Example applications with JASP. Psychon Bull Rev 25:58-76. 10.3758/s13423-017-1323-7

Walsh V. 2003. A theory of magnitude: common cortical metrics of time, space and quantity. Trends in Cognitive Sciences 7:483-488. 10.1016/j.tics.2003.09.002

Wearden JH, and Lejeune H. 2007. Scalar Properties in Human Timing: Conformity and Violations. Quarterly Journal of Experimental Psychology 61:569-587. 10.1080/17470210701282576

Wei G, and Luo J. 2010. Sport expert's motor imagery: functional imaging of professional motor skills and simple motor skills. Brain Res 1341:52-62. 10.1016/j.brainres.2009.08.014

Wiener M, Turkeltaub P, and Coslett HB. 2010. The image of time: a voxel-wise meta-analysis. Neuroimage 49:1728-1740. 10.1016/j.neuroimage.2009.09.064

Yarrow K, Brown P, and Krakauer JW. 2009. Inside the brain of an elite athlete: the neural processes that support high achievement in sports. Nat Rev Neurosci 10:585-596. 10.1038/nrn2672

You Y, Ma Y, Ji Z, Meng F, Li A, and Zhang C. 2018. Unconscious response inhibition differences between table tennis athletes and non-athletes. PeerJ 6:e5548. $10.7717 /$ peerj.5548 


\section{Figure 1}

The sample size determination process

DV1 = Dependent variable 1, DV2 $=$ dependent variable 2, DV3 $=$ dependent variable 3, DV4 $=$ dependent variable 4 . The $F$ values from previous studies were provided to calculate the partial eta square (partial $\eta^{2}$ ). Then, half the magnitude of the partial $\eta^{2}$ was translated into effect size $f$, which is needed to calculate the sample size in G*Power. Partial $\eta^{2}=0.030$ was chosen to determine the sample size for unexplored effects (DV4)

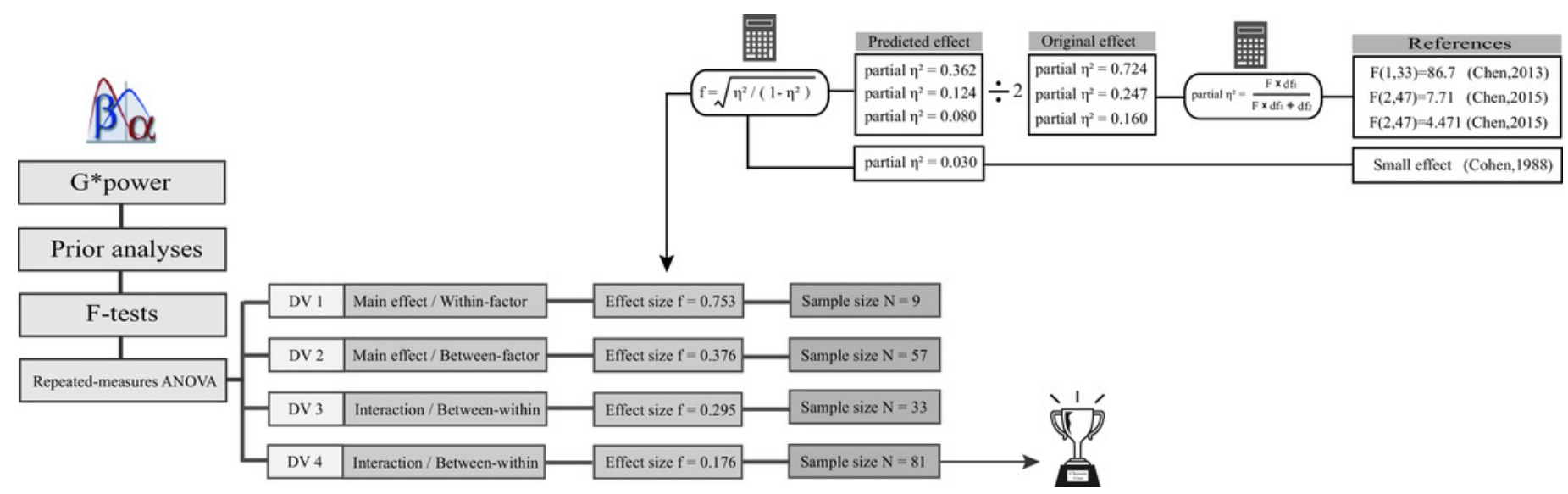


Figure 2

Temporal duration reproduction task

A sequence within a single trial of the temporal duration reproduction task in this experiment

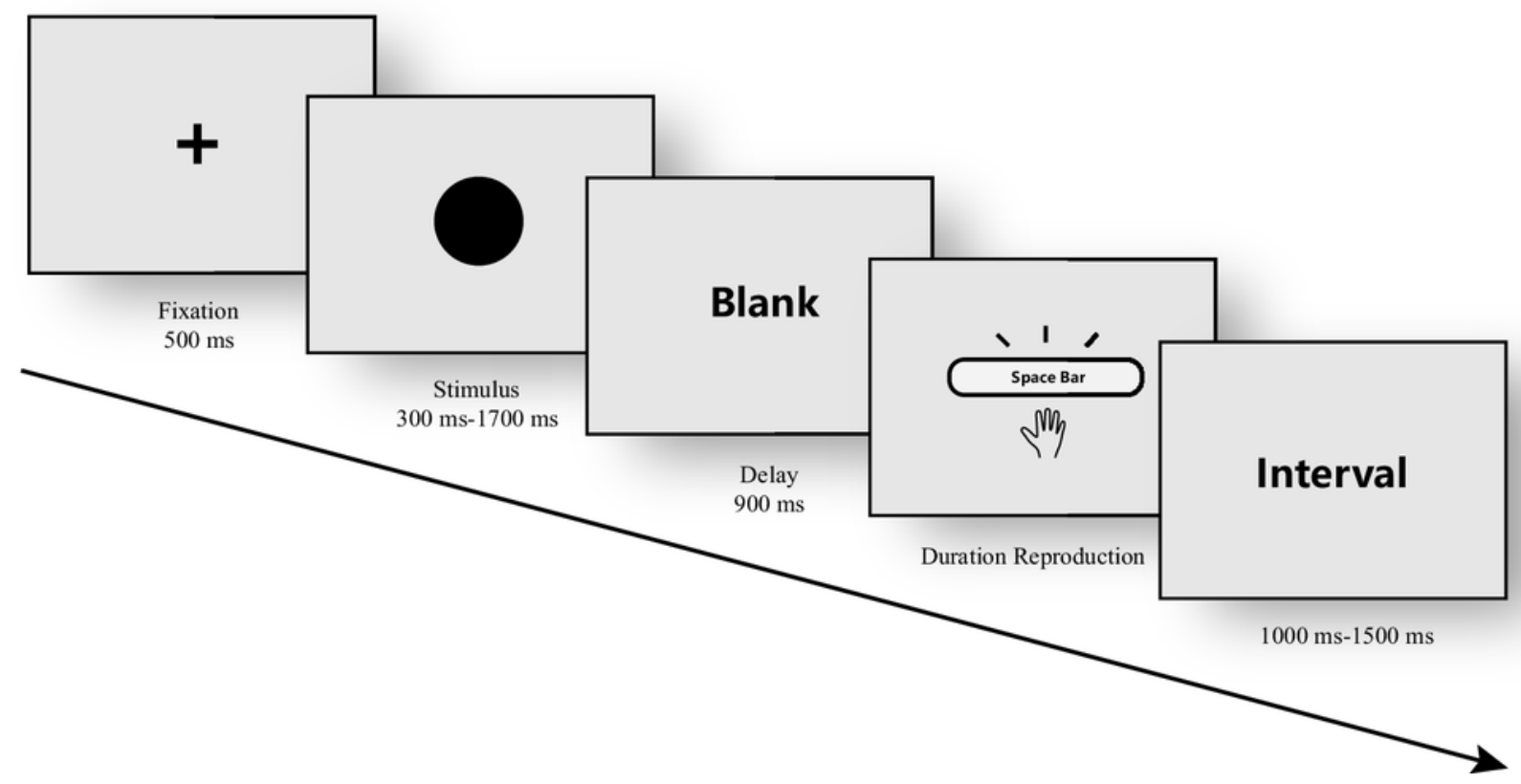


Figure 3

All stimuli in the task

(A) Stimuli in the general figure condition; (B) stimuli in the diving figure condition

A

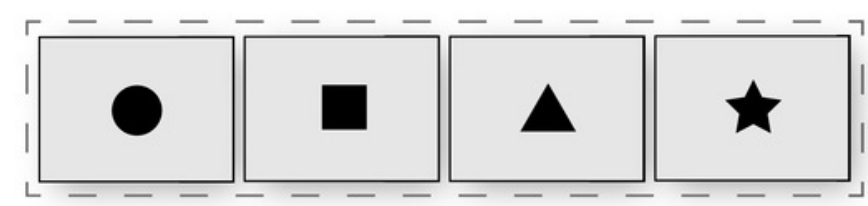

B

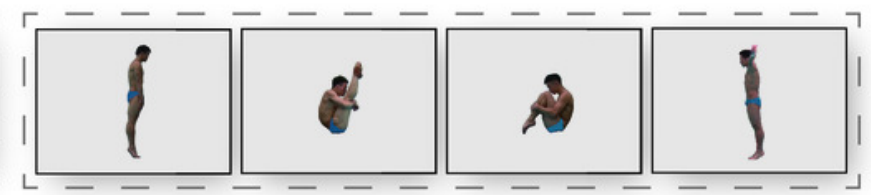


Figure 4

The experimental process

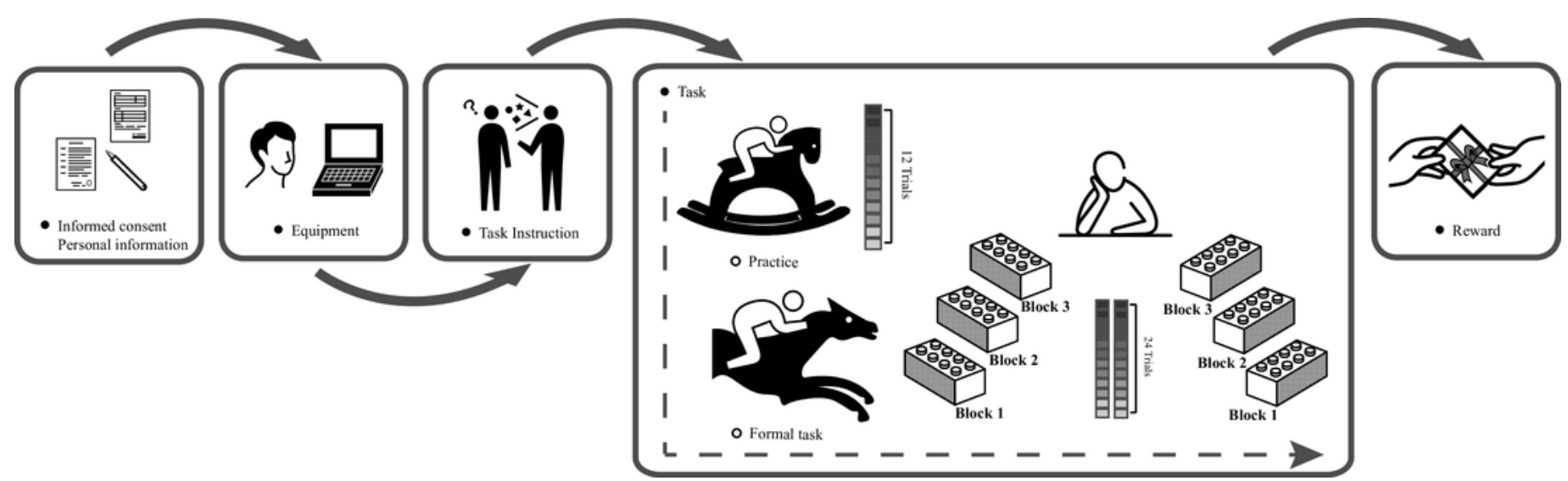


Figure 5

Reproduction duration in the three groups during the task

(A-C) The raw data with the distribution for the participants from different groups are provided via raincloud plots. The error bar indicates the $95 \% \mathrm{Cl}$ of the mean; (D-F)

Comparisons between different groups under different conditions (i.e., subsecond time range and suprasecond time range) are also presented

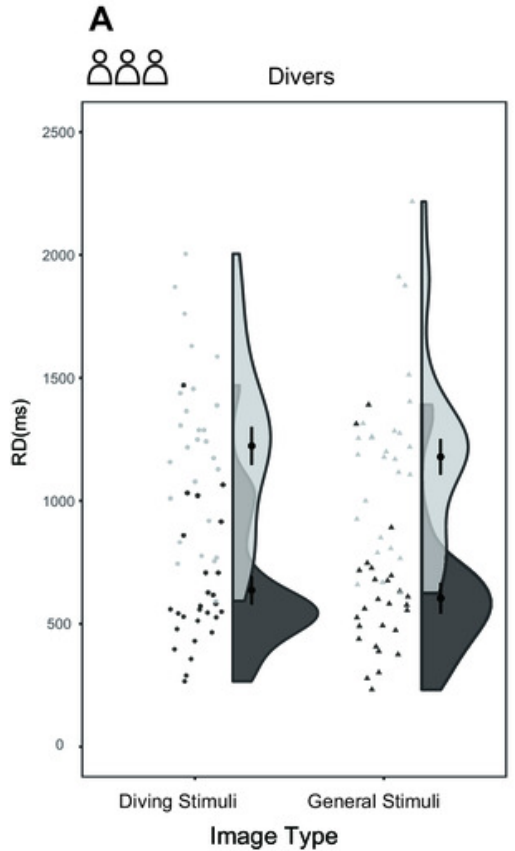

D

Interaction

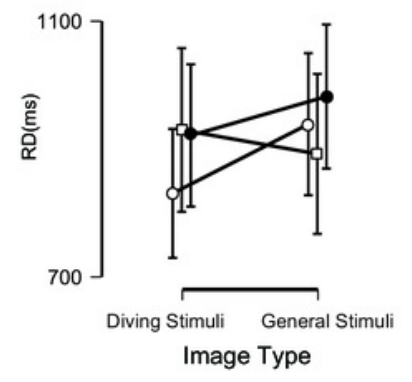

B

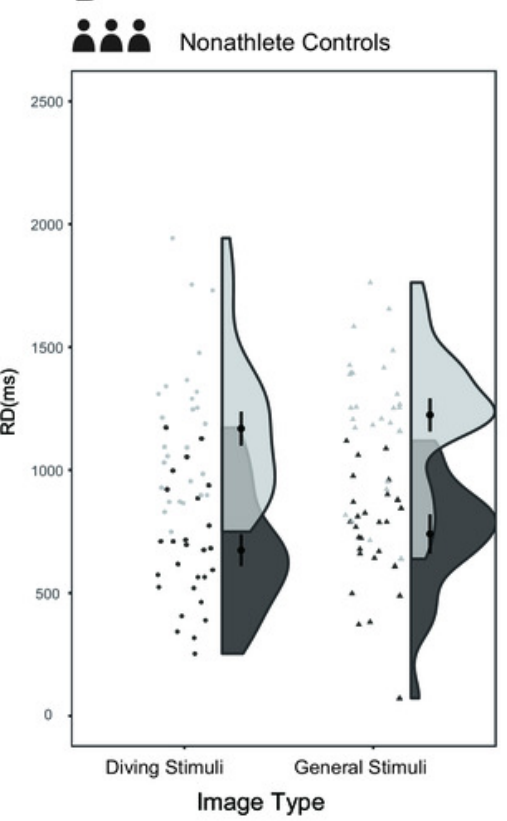

E

Time Range: Subsecond

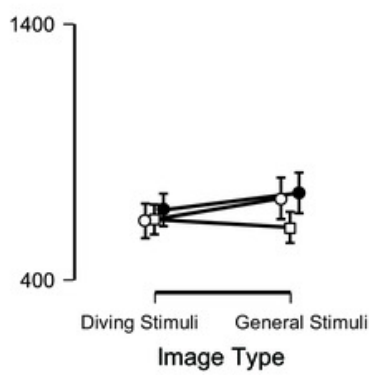

C

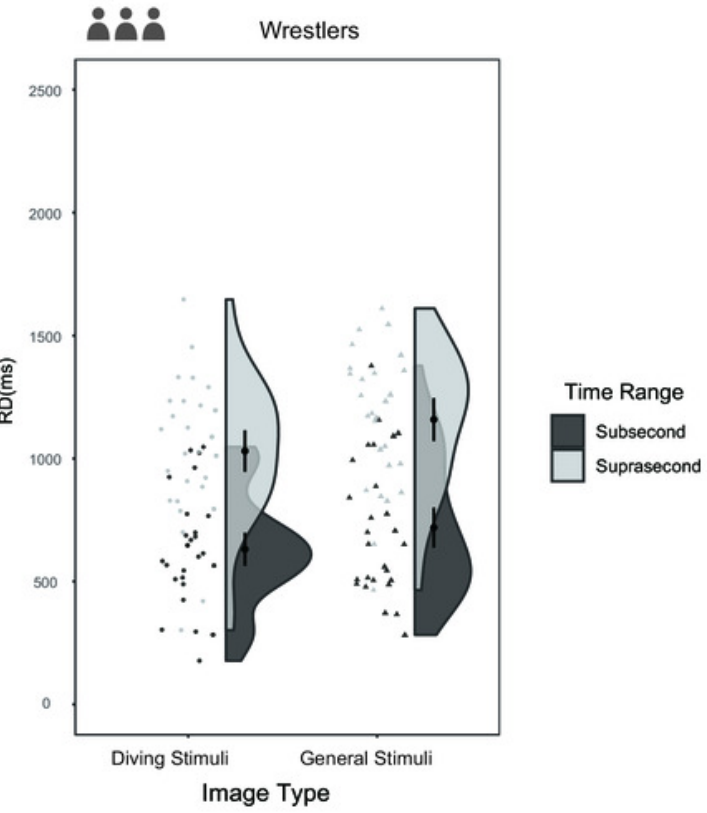

$\mathbf{F}$

Time Range: Suprasecond

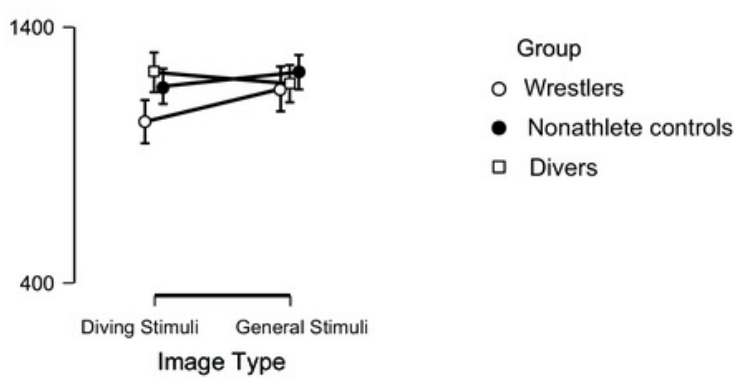


Figure 6

Reproduction duration differences in the three groups during the task

(A-C) The raw data with the distribution for the participants from different groups ; (D-E)

Comparisons between different groups under different conditions

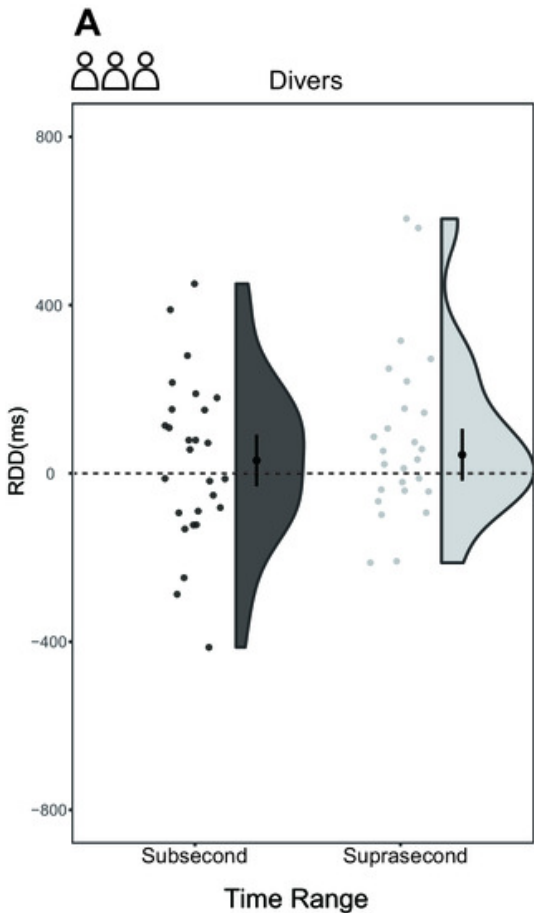

D

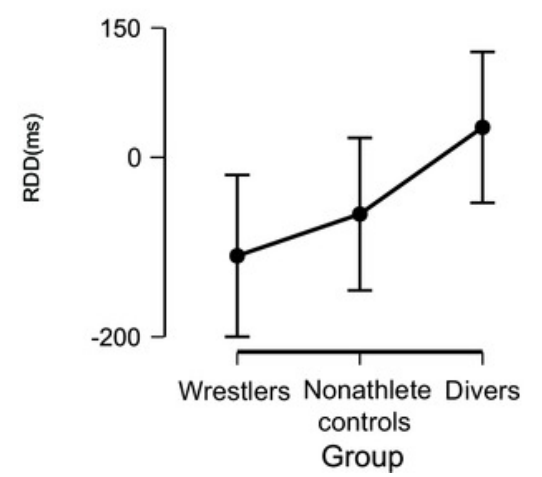

B

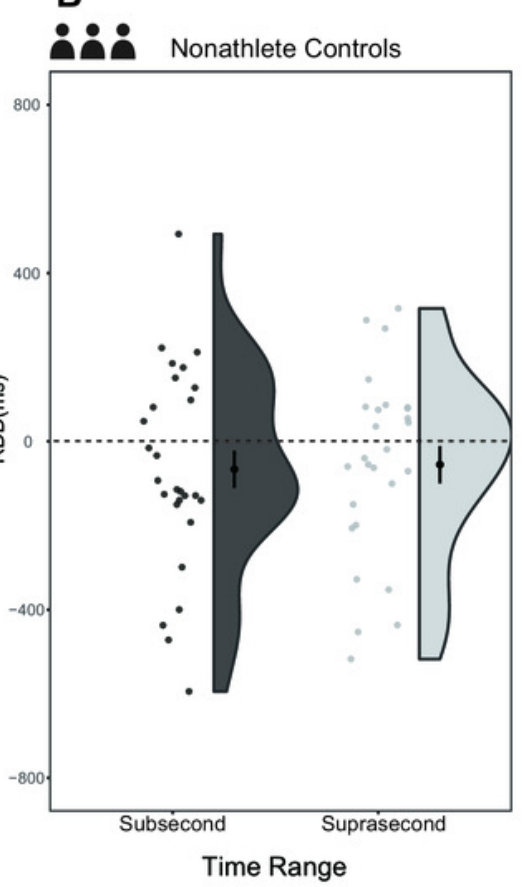

E

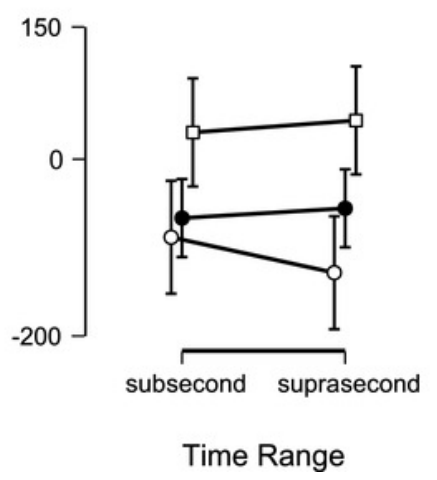

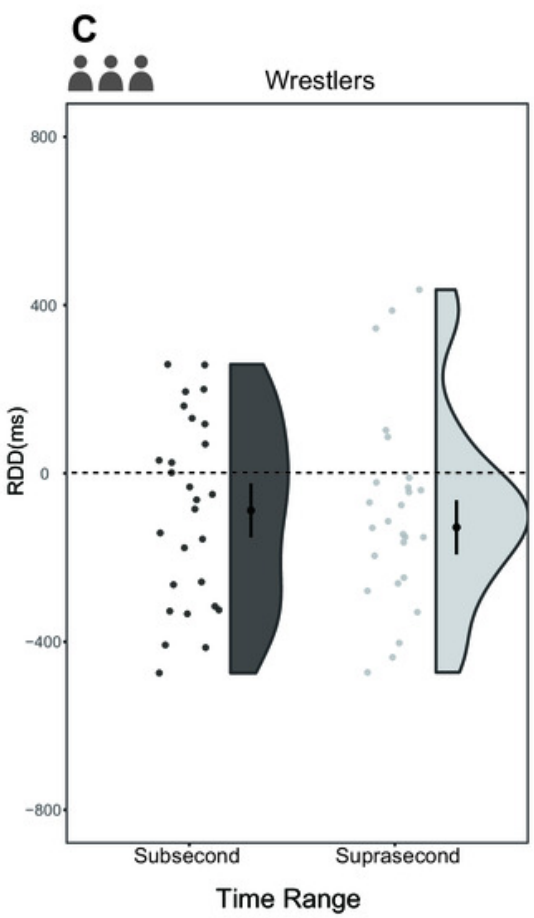

Group

○ Wrestlers

- Nonathlete controls

$\square$ Divers 
Figure 7

The correlation between the RDD and years of diving training of the divers

The lines in this plot indicate the correlation between variables based on the least squares method under different conditions (subsecond and suprasecond) 


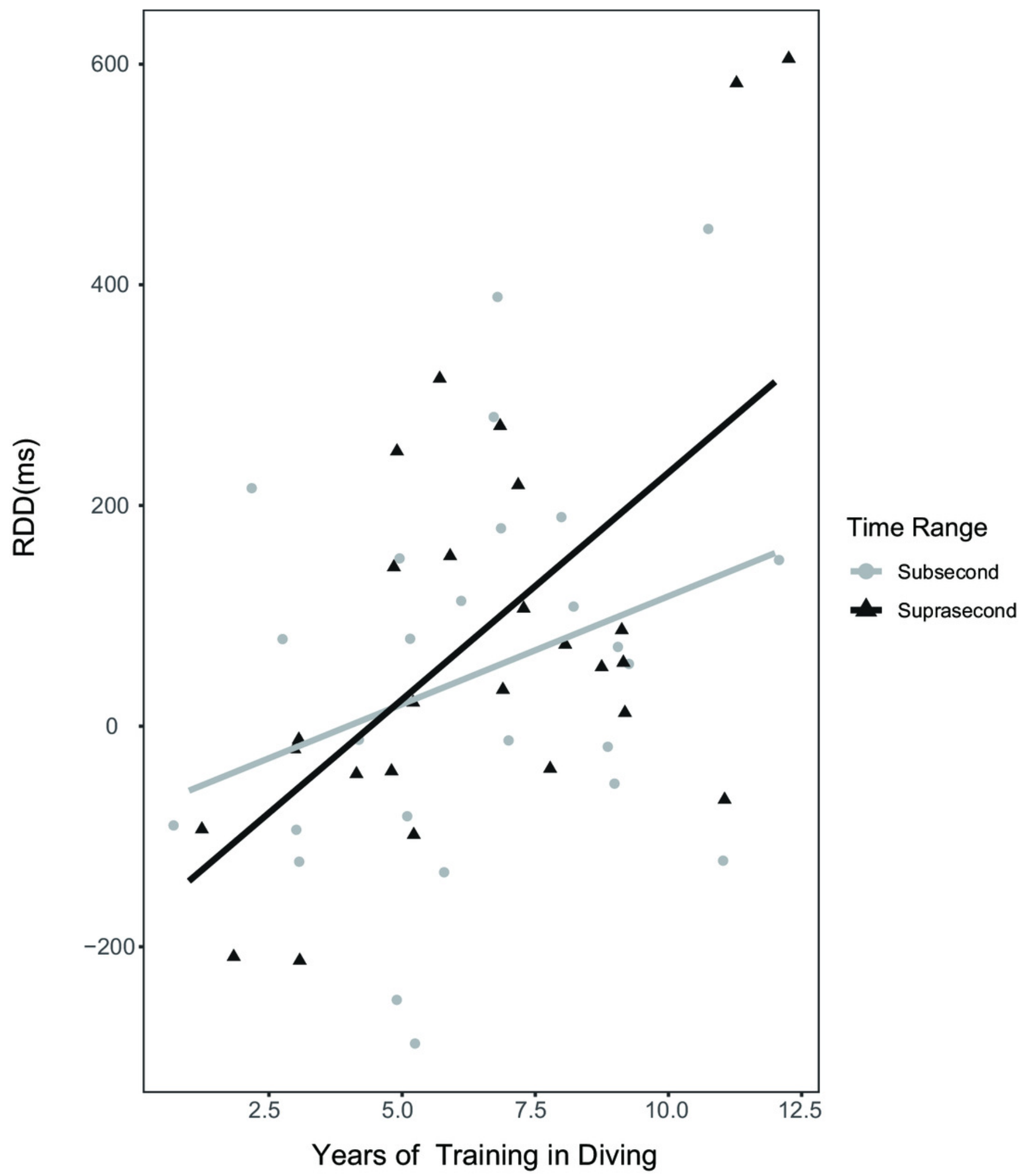


Figure 8

Reproduction duration bias in the three groups during the task

(A-C) The raw data with the distribution for the participants from different groups; (D) The interaction of group and time range;(E-F) Comparisons between different groups under different conditions
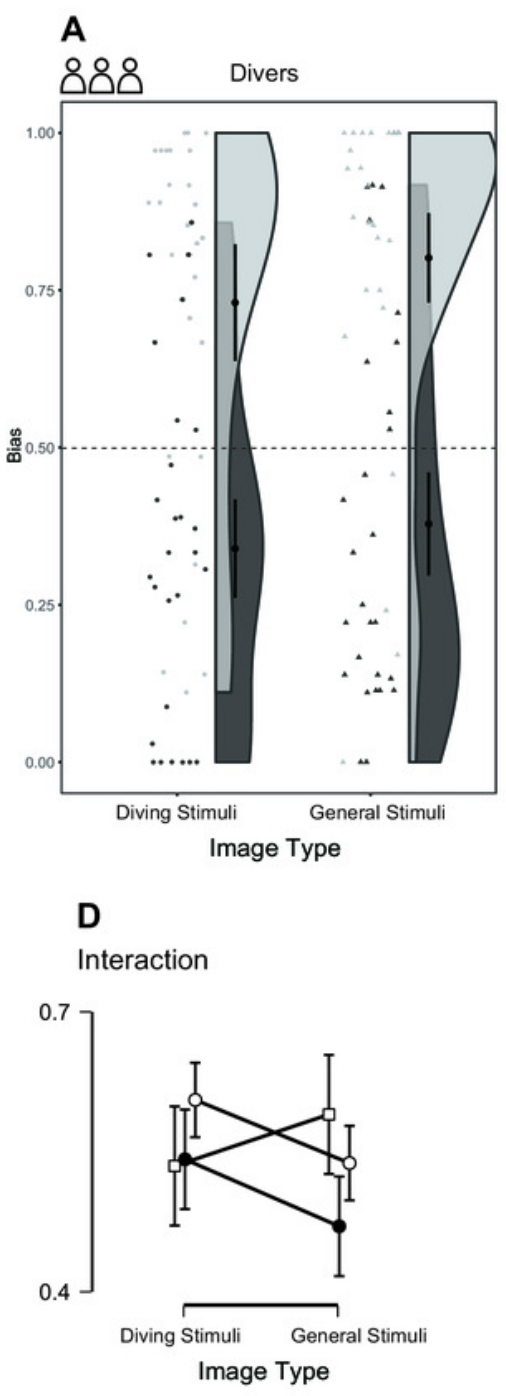

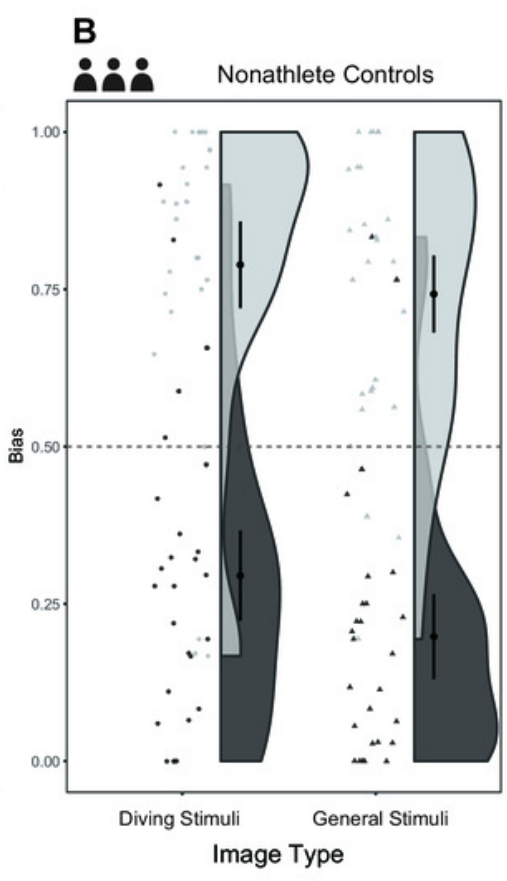

E

Time Range: Subsecond

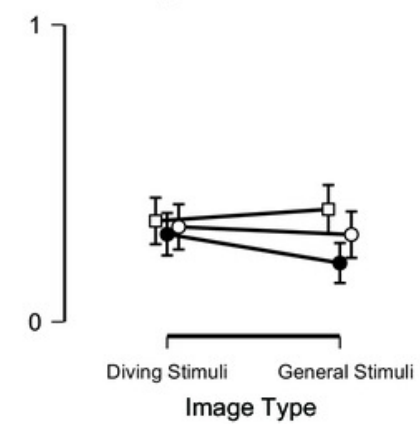

C

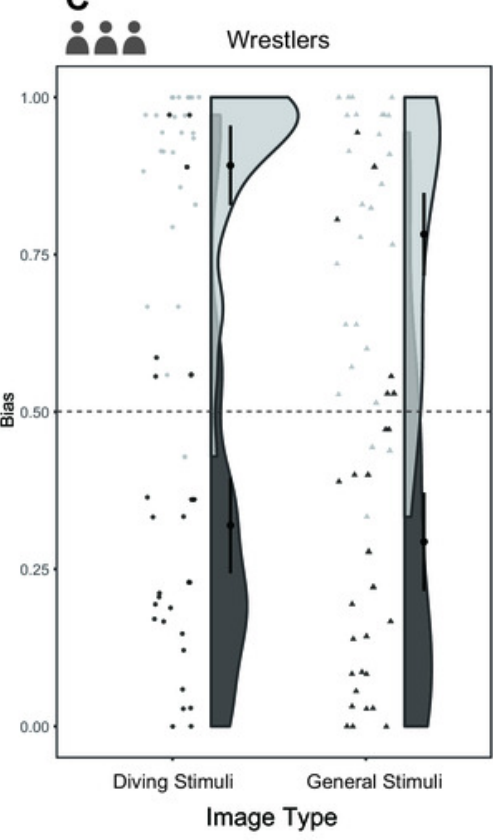

$\mathbf{F}$

Time Range: Suprasecond

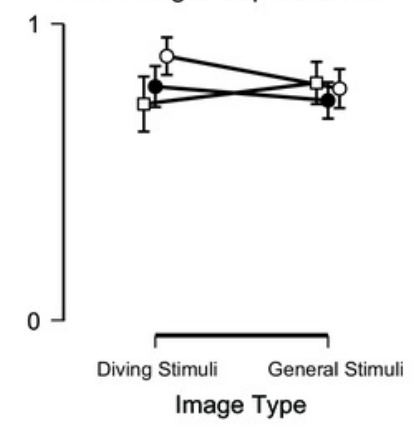

Time Range

Subsecond

Suprasecond
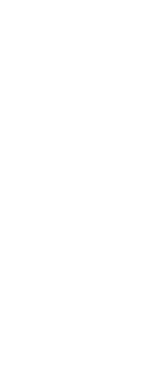

Group

○ Wrestlers

- Nonathlete controls

ㅁivers 
Figure 9

Reproduction duration CV in the three groups during the task

(A-C) The raw data with the distribution for the participants from different groups; (D) The comparison between different time range; (E-F) Comparisons between different groups under different conditions
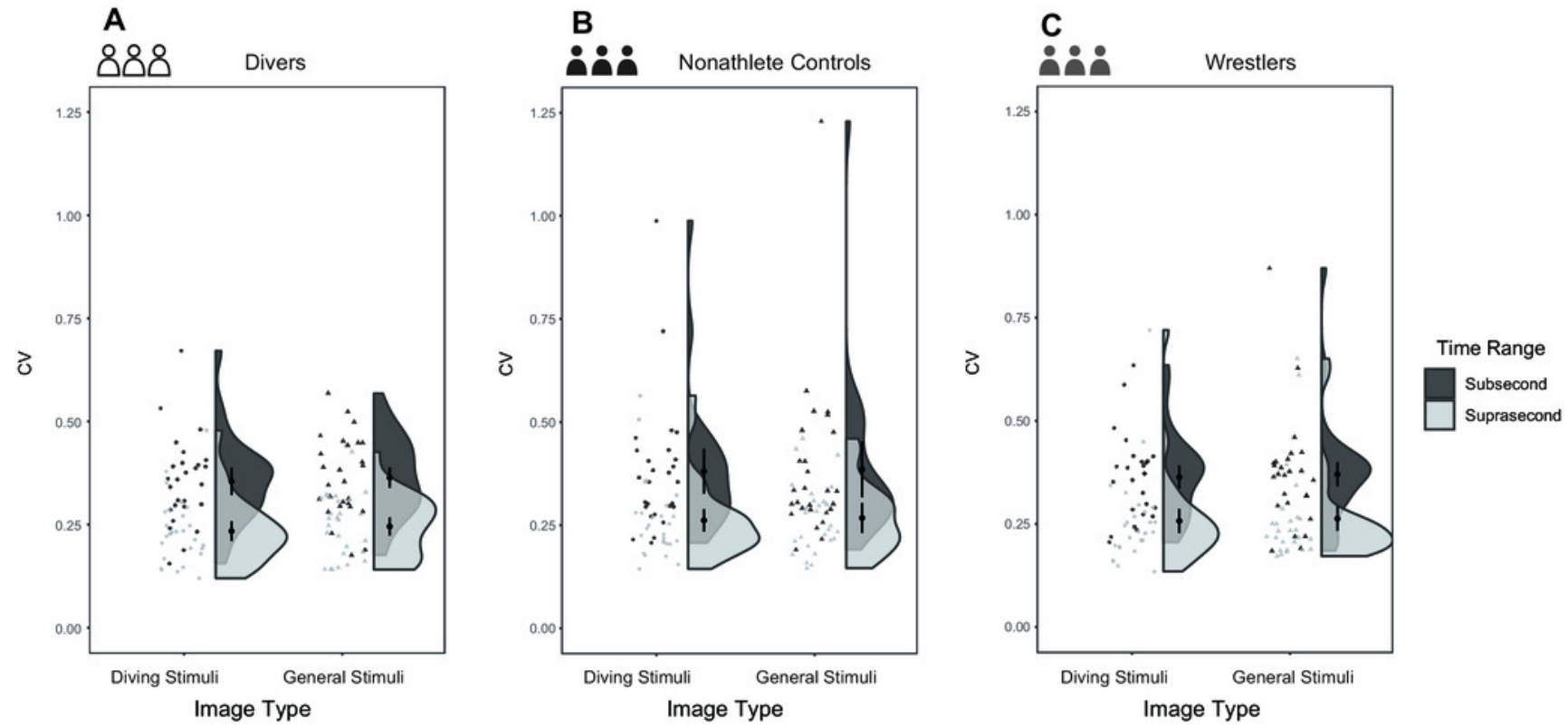

D
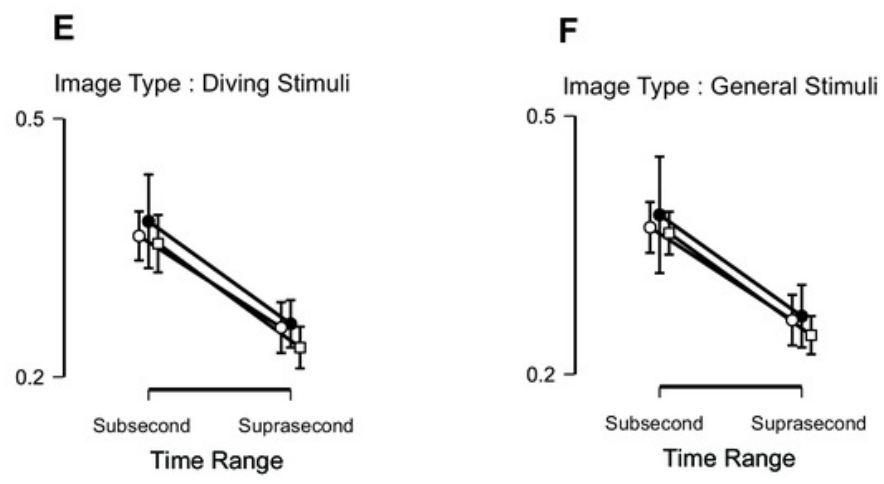

Group

- Wrestlers

- Nonathlete controls

$\square$ Divers 
Figure 10

Reproduction duration $\mathrm{AE}$ ratio in the three groups during the current task

(A-C) The raw data with the distribution for the participants from different groups; (D) The comparison between different time range ; $(\mathrm{E}-\mathrm{F})$ Comparisons between different groups under different conditions

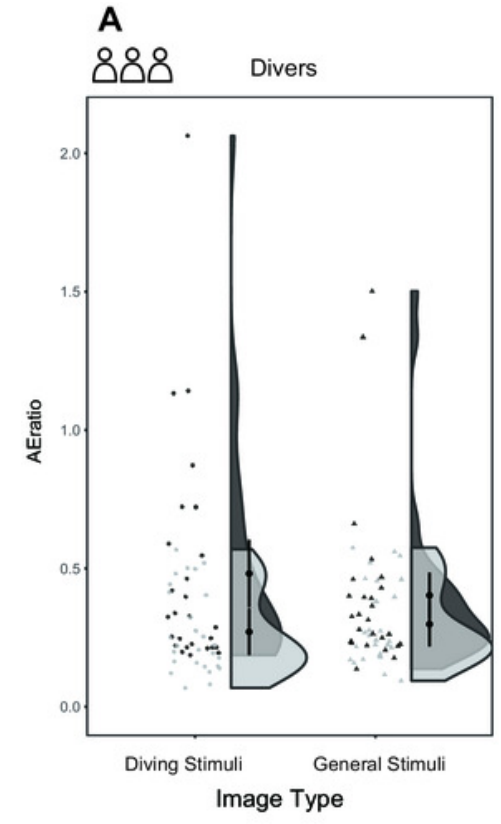

D

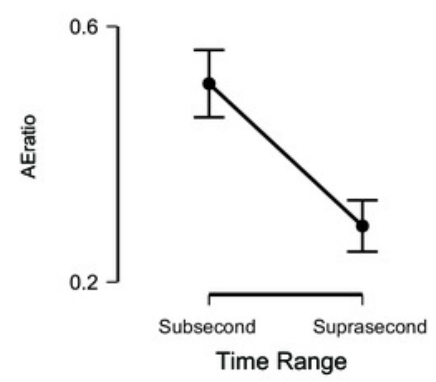

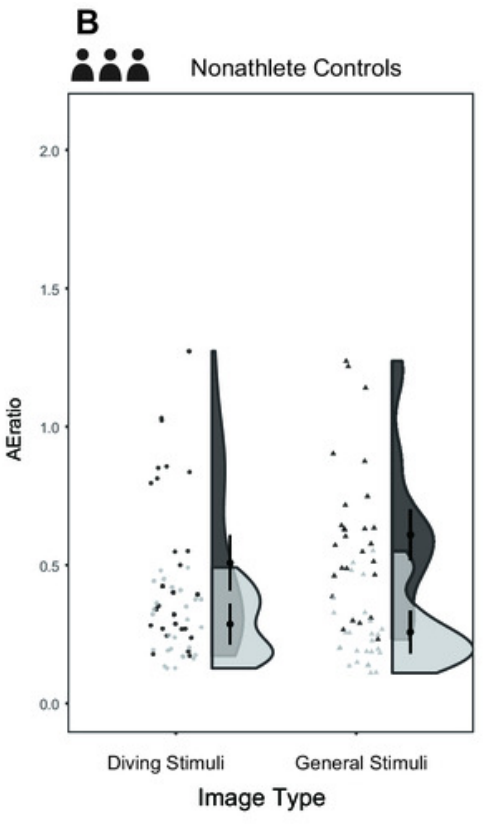

$$
\text { E }
$$$$
\text { Image Type : Diving stimuli }
$$

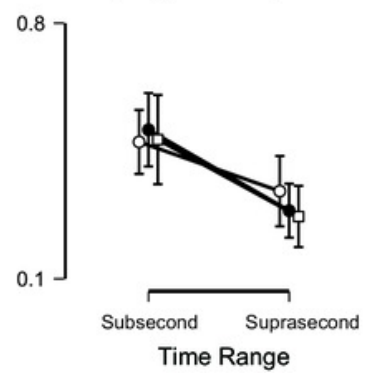

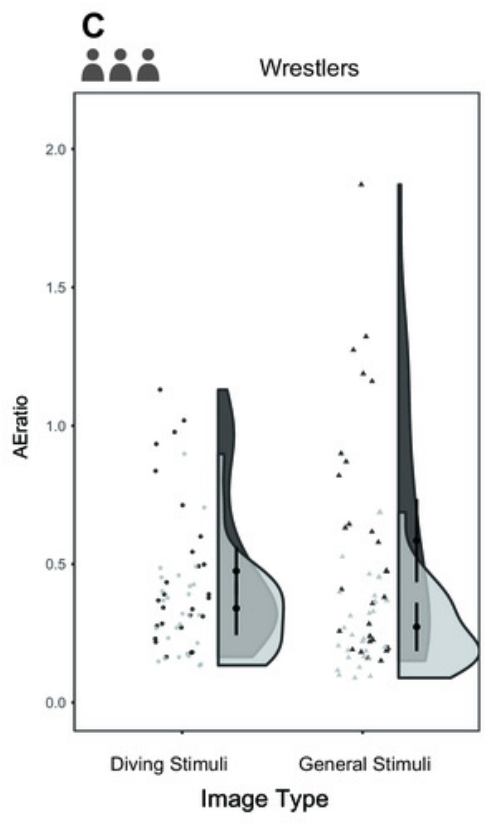
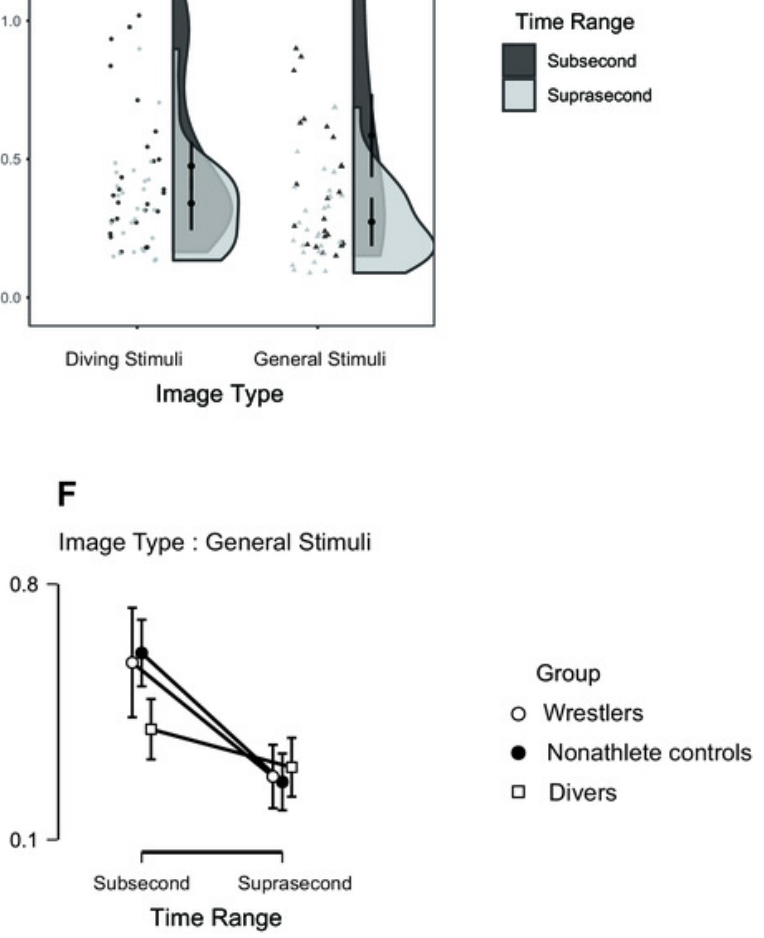
Figure 11

The analysis for effect of years of training difference between divers and wrestlers

(A-B) The correlations between years of training and RD for diving figures in wrestlers and divers; (C-E) The correlations between years of training and RDD for wrestlers and divers; (FG) Comparison between divers and wrestlers with compatible years of training (error bar= mean $\pm 95 \% \mathrm{Cl})$
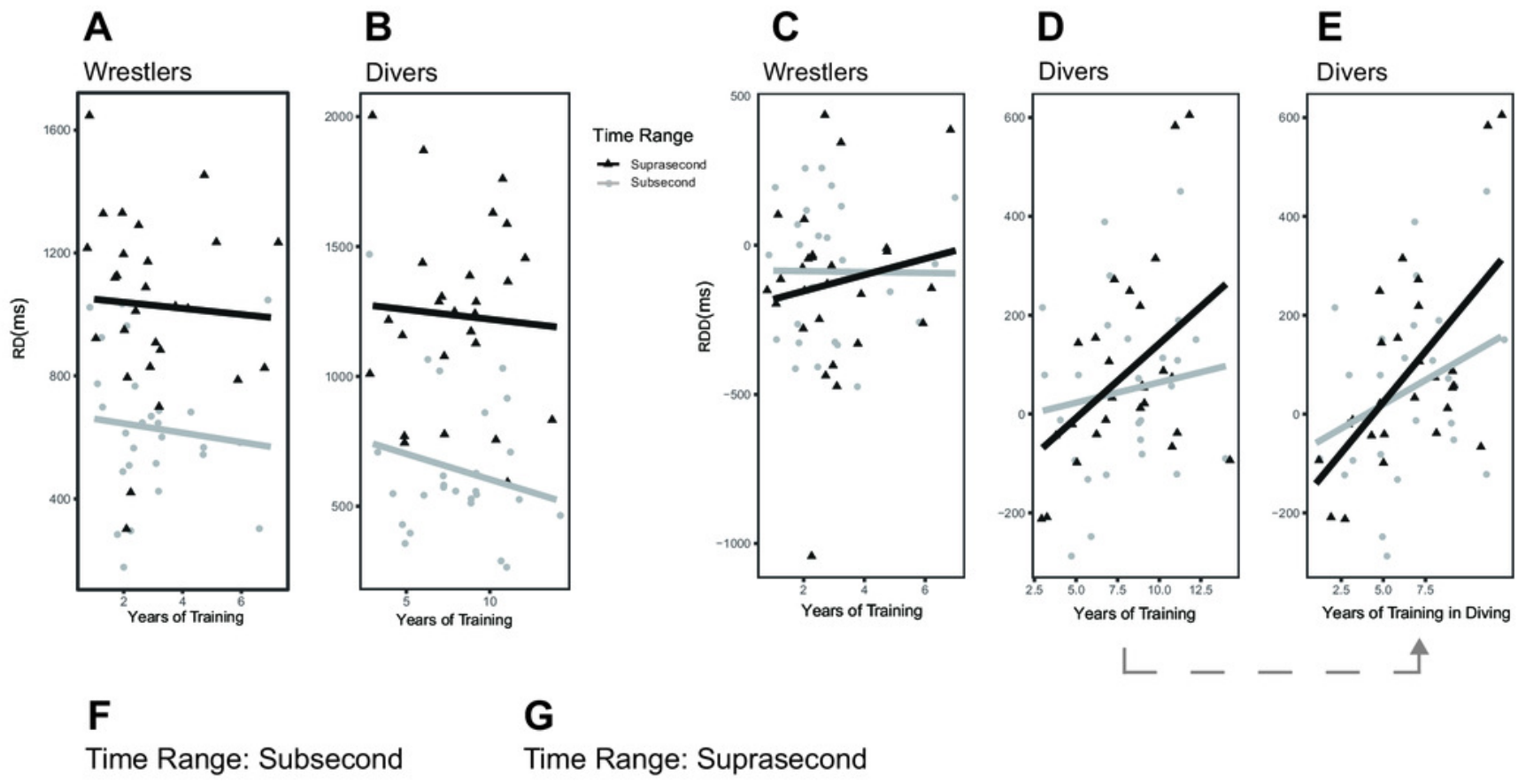

G

Time Range: Suprasecond
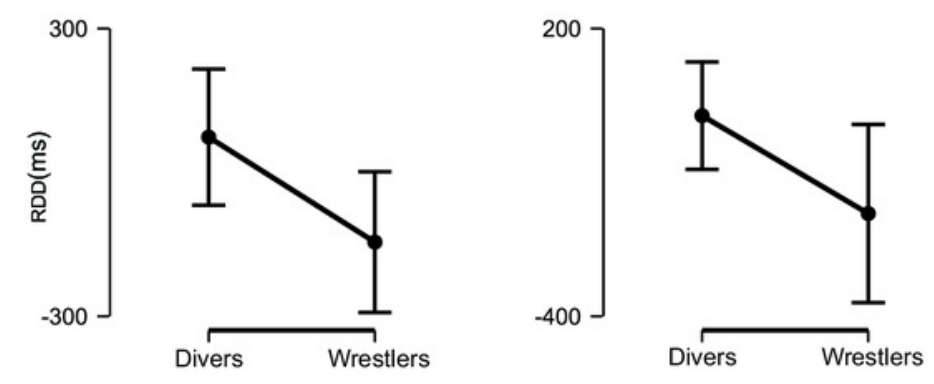